\title{
Money and Status: How Best to Incentivize Work*
}

\author{
Pradeep Dubey ${ }^{\dagger}$ and John Geanakoplos ${ }^{\ddagger}$
}

27 May 2016

\begin{abstract}
Status is greatly valued in the real world, yet it has not received much attention from economic theorists. We examine how the owner of a firm can best combine money and status to get her employees to work hard for the least total cost. We find that she should motivate workers of low skill mostly by status and high skill mostly by money. Moreover, she should do so by using a small number of titles and wage levels. This often results in star wages to the elite performers. By analogy, the governance of a society should pay special attention to the status concerns of ordinary citizens, which may often be accomplished by reinforcing suitable social norms.

Keywords: Status, Incentives, Wages
\end{abstract}

JEL Classification: C70, I20, I30, I33

\section{Introduction}

Man is moved by the desire for status. Kings wage war for glory, soldiers give their lives for honor, and gangsters take lives for respect. Professors publish for prestige. Children obey to win praise. Students study to get good grades, and sometimes protest to assert their dignity. ${ }^{1}$ Athletes train to win medals, and games are played for the thrill of victory. Donors give more when it gets them publicity. Corporate executives work hard for promotions. Military officers strive for titles. Movie stars seek fame. Everybody wants recognition, and even the ordinary man values his reputation.

Status appears in the language with such frequency and in so many guises, that it must be important. Yet in standard economic theory, status is nearly always ignored,

\footnotetext{
${ }^{*}$ This is a revision, with a slightly altered title, of the second half of Dubey-Geanakoplos (2005). The authors have recently adopted the convention of alternating the order of their names.

${ }^{\dagger}$ Center for Game Theory in Economics, SUNY, Stony Brook and Cowles Foundation, Yale University

${ }^{\ddagger}$ Cowles Foundation, Yale University, and Santa Fe Institute

${ }^{1}$ Currently across many college campuses in the United States, students are demanding safety from micro-aggressions.
} 
and the sole motivation for behavior is taken to be a desire for material consumption, the sooner and more certain the better.

In many instances status is just instrumental to getting money, suggesting that the standard theory is not wanting. ${ }^{2}$ But as the first paragraph shows, status is often sought for its own sake. ${ }^{3}$ Achilles became enraged when he was deprived of his booty, less because of its consumption value but more because of the signal it sent about his rank. Karna ${ }^{4}$ viewed honor and status as paramount, donating much of his wealth and refusing to switch to the winning side in battle, all for the glory of his reputation. Marlon Brando in "On the Waterfront" laments that in his youthful boxing days he took a dive for the money when he could instead have fought to win glory: "I coulda been a contender, I coulda been somebody". J.P. Morgan went so far as to say that money is just a way of keeping score, acquired in order to get status.

What then is status? We believe that the common theme underlying all our italicized words is: what one thinks others think of one's relative rank. Furthermore, as each of our examples indicates, rank emerges from performance, such as fighting, publishing, donating, obeying, and studying, but is not identical to it. ${ }^{5}$ What is crucial is the categorization of performance, via titles such as winners and losers, gold medalists and bronze medalists, grades $\mathrm{A}$ and $\mathrm{B}$, honorable and shameful, and so on. Status is the ranking of people induced by a ranking of the titles which label their performance. These titles (or status categories) could have evolved over time, perhaps because they encouraged behavior that made the society fitter. They could also have been created by conscious design, and nurtured by the educational system for the young, and more generally by the cultural milieu at large. In this paper we shall focus on the design aspect.

Most societies have used status to motivate their citizens. The ancient Greeks allocated honorific prizes to the best playwright, the best painter, and the best of the Achaeans. The French bury their heroes in the Pantheon. The English bestow knighthood.

The flip side of honor, indeed its negative, is shame or "losing face". The thrill of victory and the agony of defeat both create incentives for performance. Admiral Nelson's exhortation "England expects every man to do his duty" is at once a promise of honor to those who fight and shame to those who run away.

\footnotetext{
${ }^{2}$ One example of the instrumental role of money is that higher consumption may signal higher wealth and hence eligibility as a marriage partner (see e.g., Cole-Mailath-Postlewaite (1992, 1995, 1998) and Corneo-Jeanne (1998)). In tournaments, players strive for higher rank in order to get the corresponding higher monetary prize. See Lazear-Rosen (1981), Green-Stokey (1983), and Moldovanu-Sela (2001).

${ }^{3}$ To quote from James Coleman (1990, pg 130) "Differential status is universal in social systems... status, or recognition from others, has long been regarded by psychologists as a primary source of satisfaction to the self. That is, an interest in status can be regarded as being held by every person." See also Coleman (1961) for a discussion of status and its effect on school performance among adolescents.

${ }^{4}$ the tragic hero of the epic Mahabharata.

${ }^{5}$ We only consider ranks that are earned; not those which are inherited at birth, such as titles of nobility, slavery, caste, etc. (The latter, while widely prevalent in the past, are getting eroded with time, and hopefully on the way out.)
} 
In modern day labor compensation schemes, we see that many firms use status to motivate their employees. Titles, like associate, vice president, and managing director, not only fix organizational roles within a firm, but bestow prestige and rank. ${ }^{6}$ At a lower level in the hierarchy, many companies such as McDonald's and Walmart post pictures of the "employees of the month" to honor their most productive workers.

The general question we seek to answer is, how should the owner of a firm combine status and money to motivate her employees to work with the least expenditure of money? ${ }^{7}$ How many titles should she award? Should she use titles to lower all the wages, or is it better for her to use titles to lower some wages and actually raise others? Should she provide status incentives to performers at the top or at the bottom? Should she focus on honor or on shame?

To answer these questions, we introduce a mathematical approach to status, involving the owner of a firm and her employees, which we believe is novel. (Prior approaches are discussed in the next section.) We assume that the employees get utility out of their status in the firm, as well as from their wages. Status is conferred by titles with which the owner labels their performance. ${ }^{8}$ We suppose that individual performance or output is objectively measurable and observable to the owner and to the individual, but not marketable: the employee cannot sell off part of his output to another employer. Employees may have differential abilities, and they can either work or shirk. Each employee's expected output is increasing with his effort and ability, but the actual output depends also on chance. The owner cannot tell in general whether a particular output was caused by effort, ability, or luck. She can only observe the outputs of her employees, and on that basis alone must reward each of them with both a title and a wage. ${ }^{9}$ We require that rewards be merit-based in that a unilateral increase in the ouput of any employee cannot lower his reward: his wage cannot go down, and if his title earlier outranked another's, it continues to do so. ${ }^{10}$

Wages can be public or secret. When wages are public, as in state universities and government offices in the United States and many other countries, they enable or force people to compare themselves with each other. Public wages in effect create titles along with monetary compensation. In our model with public wages, an output that gets a higher wage necessarily gets a higher title, but we do allow titles to go up across outputs that are given the same wage. ${ }^{11}$

\footnotetext{
${ }^{6}$ In fact we shall abstract away from the organizational function of titles, and focus only on the status incentives created by titles.

${ }^{7}$ More generally we could introduce money and status into any non-cooperative game. This is done by augmenting the game form with monetary rewards as well as a ranking of the players, both based on the outcomes arising from the actions in the game. See Dubey-Geanakoplos (2016) forthcoming.

${ }^{8}$ Sometimes these titles become known to people outside the firm, especially for the highest ranked employees, in which case they may gain still more status utility. We discuss this external status incentive later in the paper, but for the most part, we focus on internal status.

${ }^{9}$ We actually allow the employer to award a lottery over wages and titles.

${ }^{10}$ The rewards are non-discriminatory: employees who produce the same output get the same title and wage, unless the rewards are randomized, in which case their lotteries are the same.

${ }^{11}$ We easily accomodate the case where wages alone signify title. If two titles are given the same wage, we could pay the higher title infinitesimally more if we wanted to identify titles with wages.
} 
Sometimes wages can be secret, as happens in many private institutions, such as Yale University and the Santa Fe Institute, where there is an unspoken rule that employees do not discuss their salaries. This gives the owner more flexibility, for then she can pick wages and titles completely independently of each other.

We cannot solve for the optimal wage-title schedule in general, though we can show they always exist. So we carry out our analysis in two polar extremes, with homogenous workers or with disparate workers, where exact solutions can be computed. Even here the mathematics becomes subtle. Nevertheless, there are common themes that emerge from both cases.

First, it is evident that the owner should use status as much as possible, since titles cost her nothing to bestow. Firms that ignore status will have higher wage costs and be less profitable. Money payment need not be contemplated until status incentives are exhausted.

Yet in spite of their costlessness, it is optimal for the owner to award very few titles. Even though there may be numerous levels of performance, it would be best for her not to discriminate among them too finely. She should partition them into a few broad categories and award titles based on those categories alone. Awarding more titles would reduce effort, or force her to pay higher wages to maintain the same effort. This is consistent with the remarkably small number of titles that firms award compared to the number of their employees or the range of individual performances. As was said, Walmart and McDonalds announce the employees of the month, in effect creating two titles, i.e., the elite group and the rest; but they never publicly rank all their employees from top to bottom.

The optimal wage schedule is also a step function, made up of broad wage slabs, not the steadily increasing curve that would be generated, say, by a piece rate. ${ }^{12}$ The inevitable consequence is that at the cut points of the slabs, a small increase in performance will lead to a higher title or a big jump in wage. ${ }^{13}$ Our results reflect the reality that in many bureaucracies, both in public and private spheres, employees are placed in a hierarchy consisiting of a few groups, in each of which the wage is roughly constant despite variations in productivity, and that across these groups, there are jumps in wages. Our results also suggest (though this is less well documented in practice) that the jumps in pay across groups might occur without corresponding jumps in productivity.

One reason for broad categories is that often times higher performance is achieved by luck or ability rather than effort; steadily increasing rewards for increasing outputs can reward luck or ability and penalize effort. ${ }^{14}$ A second reason is that broad cate-

\footnotetext{
${ }^{12}$ We shall often use the term "wage ladder" for this step function, with each step referred to as a "rung".

${ }^{13}$ In some cases the optimal cut must be by lottery, so that employees with the same performance will get radically different wages and titles.

${ }^{14}$ It would be naive to assume that higher output is always a sign of higher skill and better luck and more effort. This is particularly true when effort must be devoted to mastering multiple tasks. The low effort employee only gets to the first task; the high effort employee gets to many tasks, but then is slightly less good at the first task. Imagine a student who studies just one problem and can get it right $80 \%$ of the time, while the high effort student studies both semesters and can get each of two problems right $60 \%$ of the time. The shirker has probabilities $(.2, .8,0)$ of getting 0,1 ,
} 
gories generate higher status incentives for employees with lower ability by enabling those who work hard to come equal in title with workers of higher ability, a little like handicaps do. (The difference is that handicaps discriminate between people, giving a boost say to minority candidates, whereas our categories are anonymous). This becomes important if it is optimal to use status to incentivize employees of lower skill.

Another theme of our analysis is that the owner should incentivize high performers mostly by money and low performers as much as possible by status. This sounds counterintuitive, since by hypothesis the highest status is earned by the highest performance. But there is no contradiction, since status incentive derives from the change in status that effort can bring, not from the level of status one winds up with.

The deeper explanation is that with meritocratic pay schedules, a wage hike between low and middling performance forces a higher wage for all superior performance, and is therefore very expensive, while the same wage hike between excellent and the best performance does not necessitate any further raises. Thus the more that status incentives can substitute for pay hikes at low performance levels, the less the total wage bill.

This theme comes through very starkly in both the homogeneous and disparate cases. In the disparate case with secret wages, it is optimal to award just two titles (member vs non-member) so that all the status incentive goes to the very lowest ability group. With public wages it is optimal, in both the homogeneous and disparate cases, to award exorbitant wages to a tiny elite of top performers while using titles as much as possible to motivate the others. ${ }^{15}$ This is consistent with the high pay hikes we see at the top of the corporate ladder for CEOs. Again our analysis shows that this jump at the very top may not be accompanied by a jump in productivity.

Finally we show that as status becomes more valued by employees, the optimal wage-title schedule becomes increasingly "star-like": the percentage hike escalates more rapidly as we go up the wage ladder.

The analysis we have applied to a firm and its owner may be reinterpreted more broadly in terms of a society and its governance. Those in power should think carefully about how to shape the status categories, through a judicious designation of titles. They can use cash to induce citizens to obey. They can also coerce citizens through the penal system. But status is a third powerful motivator that they would ignore at their own peril. ${ }^{16}$ Status undoubtedly played a critical social role before money was invented, and even in modern times, money payment need not be

or 2 correct answers, while the high effort student has probabilities $(.16 ., 48, .36)$. (In the language we shall shortly introduce, the worker stochastically dominates the shirker, but does not uniformly stochastically dominate him.) The optimal title partition or wage schedule will turn out to be \{ $\{0,1\},\{2\}\}$ in which all the rewards are reserved only for getting both answers right.

${ }^{15} \mathrm{In}$ fact, when employees have risk neutral preferences for money, it is optimal to award an arbitrarily high wage with arbitrarily low probability to the highest performances. Existence of an optimum requires the assumption of a maximum wage beyond which the firm cannot pay.

${ }^{16}$ The recent unrest at many college campuses led by students of color protesting miccro-aggressions and unsafe feelings appears at bottom to be a demand for status. 
contemplated until status incentives are exhausted.

Many of the examples we gave at the beginning of the introduction involved status rewards for top end performances. What are the status symbols for the ordinary folk that the theory suggests should be even more important? We submit that many social norms are enforced not by monetary rewards, or jail for people who flout them, but by the loss of status that attends those who break them. Most citizens want the status of being known as decent or honest even if there is no crime in being indecent, and even if they are old and beyond the time when a reputation for honesty will help them get a business partner.

\section{$1.1 \quad$ The Model}

The owner of the firm defines a wage-title schedule, mapping the vector of outputs $x=\left(x_{1}, \ldots, x_{N}\right) \in \mathbb{R}_{+}^{N}$ of her $N$ employees to their wages $w(x)=\left(w_{1}, \ldots, w_{N}\right)$ and titles $t(x)=\left(t_{1}, \ldots, t_{N}\right)$, where each title $t_{i}$ comes from a set that has a total order $>.{ }^{17}$ For the most part we restrict attention to absolute reward schedules, in which the owner partitions the output space $\mathbb{R}_{+}$into consecutive intervals. This partition serves as the common yardstick for every employee's performance. Each interval is labeled with a title and a wage, and in keeping with the meritocracy criterion, wages and titles cannot go down as the intervals go up.

The owner could have considered reward schedules where the wage and title of one worker depend on the output of the others. The most common would be a relative reward schedule in which the top $r_{1}$ outputs are given the highest award, the next $r_{2}$ the second highest and so on. We prove in Section 4 that an optimal absolute schedule is better for the owner than any relative schedule, so we may ignore relative schedules altogether. But there are still more general schedules, beyond the absolute and the relative, that we do not investigate.

The wage-title schedule is known to all the employees. If employee $n$ works (effort $\left.e_{n}=1\right)$ he produces a random output, and if he shirks $\left(e_{n}=0\right)$ he produces a different random output with a lower expectation, but without incurring the disutility $d_{n}$ of working. Based on the realizations $x=\left(x_{1}, \ldots, x_{N}\right)$ of outputs, each employee $n$ obtains utility

$$
u^{n}\left(w, t, e_{n}\right)=u_{n}\left(w_{n}\right)+\sigma_{n}\left[\#\left\{j: t_{n}>t_{j}\right\}-\#\left\{j: t_{j}>t_{n}\right\}\right]-e_{n} d_{n}
$$

Every employee $n$ gains $\sigma_{n}$ utiles for each person he outranks and loses $\sigma_{n}$ utiles for each person who outranks him. ${ }^{18}$ There is some recent evidence that such utilities

\footnotetext{
${ }^{17}$ It would be interesting to extend the model to multidimensional outputs and partially ordered titles.

${ }^{18}$ An alternative would be to suppose that status utility comes from being the top dog, or more generally, is increasing and stricly convex in the number of people an employee outranks. Strict convexity might arise if higher rank gives higher visibility to the outside world; for example, only the CEO might enjoy media attention outside the firm. In this paper we are ruling this out, imagining a closed world in which status utility is derived from the acknowledgment of superior rank by the other employees; the owner alone is the public face of the company. However our framework can accommodate such non-linear status utilities, and in particular the existence of optimal wage-title
} 
are quite prevalent in reality. ${ }^{19}$

We suppose that utility $u_{n}$ for money is increasing and concave. Our results are most powerful in the risk neutral case when $u_{n}$ is linear, for when there is diminishing marginal utility, it is very difficult to further motivate highly paid employees with still more money.

The wage-title schedule defines a strategic game among the employees. The goal of the owner is to design a schedule with the cheapest wage bill such that there is a Nash equilibrium in which everyone works.

In Section 2 we consider employees of homogeneous (i.e. ex ante identical) abilities, and in Section 3 we consider employees with disparate abilities. ${ }^{20}$ In both cases we solve for the optimal title schedule when wages cannot be paid, the optimal wage schedule when titles cannot be awarded, and finally the optimal wage-title schedule. For homogeneous workers it turns out that the optimal title schedule remains the same after it becomes possible to pay public wages as well (and thus there is nothing to be gained by keeping wages secret). However, for disparate workers, the optimal title schedule must be adjusted once public wages are introduced (and thus the owner can exploit secret wages). In both the homogeneous and disparate cases, increasing performance near the low end brings more status but not much more wages, while at the high end, there are huge money bonuses for star performers. In both cases, this "star-like" quality of the wage schedule gets more pronounced as status becomes more important.

The general case of employees with overlapping abilities - which lies in between our extremes - is no doubt important, and our framework makes it clear that optimal wage-title schedules exist in this case as well, though their precise structure is not investigated here.

Furthermore, for the most part we assume complete information, i.e. each employee knows not only his own ability, but also the population distribution of abilities of his rivals. In Section 5 we show that our results remain essentially intact with incomplete information where each employee has a probability distribution on the abilities of his rivals but does not know their actual realizations.

In the future we hope to investigate which lessons persist if outputs depend to some extent on cooperation between the employees that might be compromised by jealousy, or if there is competition between firms to hire workers, or if performances and rewards occur repeatedly in a dynamic framework.

schedules is not compromised, as we shall show in a sequel paper. The planner will adapt his rewards depending on how his employees perceive status.

${ }^{19}$ See for example the article "Does Wage Rank Affect Employees Well-being?" by Brown, Gardner, Oswald, and Qian (2008).

${ }^{20}$ These two cases were also the center of attention in Dubey-Geanakoplos (2010), in the scenario where wages were not present, and students were rewarded solely by titles/grades based on their exam scores. 


\subsection{Related Literature}

Status has been discussed by many authors from various points of view. There is a large literature whose pioneers include Weber (1922) and especially Veblen (1899), who famously introduced conspicuous consumption, i.e., the idea that people strive to consume more than others partly for the sake of higher status.

A major distinction turns on cardinal versus ordinal utility. One strand of the literaure adopts a cardinal approach which makes utility depend on the difference between an individual's wage/consumption and others' consumption (see, e.g., Duesenberry (1949), Pollak (1976), Fehr-Schmidt (1999), and Dubey-GeanakoplosHaimanko (2013)). The ordinal approach makes utility depend on the individual's rank in the distribution of consumption (see, e.g., Frank (1985), Robson (1992), Direr (2001), and Hopkins-Kornienko (2004)). See Auriol-Renault (2008) for a recent survey of the literature, including different approaches to the modeling of status. Our model is in the ordinal tradition.

Another strand starts with the hypothesis that status increases the marginal utility of consumption. Friedman-Savage (1948) and Friedman (1953) invoke this hypothesis to study attitudes toward risk taking. Becker et al (2005) use the same hypothesis to explain how inequality might arise in a Pareto efficient equilibrium. In our model, we eschew this hypothesis: status does not affect the marginal utility of consumption.

The novelty of our approach is twofold. First we link status with performance, rather than with consumption. Goode (1979) explicitly did the same, but without any formal mathematical model. Second, we partition performance into broad status categories that are chosen by a planner. Thus large gains in performance may not necessarily bring more status, in contrast to the earlier literatures on cardinal and ordinal status, where more (consumption) always gives higher status.

We introduced our model of status in an unpublished Cowles Foundation working paper Dubey-Geanakoplos (2005). Moldevanu, Sela, and Shi (2007) considered an interesting variation of our (2005) model called "Contests for Status". They used the same utility function for money and status, and they also envisioned a planner creating status through performance categories as we did. There are, however, several differences between the two models, and their perspectives are complementary. They allow for a continuum of effort levels, where we have only two. Our owner cannot disentangle effort, skill, and luck, whereas theirs always can (since they assume effort equals output). Their status categories are based on relative performance, whereas ours are based on absolute performance; in our context, absolute always outperforms relative from the owner's (planner's) point of view. ${ }^{21}$

\footnotetext{
${ }^{21} \mathrm{~A}$ longer catalogue of differences is as follows. Output in their model is deterministic, while ours permits randomness (which we call luck). In their model all agents have identical productive skills, whereas in our disparate model skills are heterogeneous. In their model disutilities of effort are ex post heterogeneous, though ex ante identical, whereas in our disparate model they are both ex ante and ex post heterogeneous. In their model effort is observable to the owner, whereas in our homogeneous skill case, the owner cannot disentangle effort from luck. In their model, there is incomplete information among the employees about each others' disutilities of effort, whereas we mostly focus on complete information. They restrict attention to performance on a relative scale,
} 


\section{Homogeneous Employees}

We first consider the case of $N$ homogeneous, i.e. ex ante identical, employees. For simplicity suppose that the possible outputs lie in a finite ${ }^{22}$ set $Q \subset \mathbb{R}_{+}$, with maximum $x_{\max }$ and minimum $x_{\min }$. If any employee works, his output is a random variable $X$ with density $f$ on $Q$, and if he shirks it is a random variable $Y$ with density $g$ on $Q$. For any subset $A \subset Q$, let $f(A)=\sum_{x \in A} f(x)$ and $g(A)=\sum_{x \in A} g(x)$. We suppose that the output of each employee is statistically independent of the others' outputs regardless of their effort levels. ${ }^{23}$ The disutility for switching from shirk to work is denoted by $d_{n}>0$ for all employees $n$. Since employees are ex ante identical, we take $\sigma_{n}=\sigma, u_{n}=u, d_{n}=d$ for all employees $n$.

We make the productivity assumption that the worker on average produces more output than the shirker:

$$
\sum_{x \in Q} f(x) x>\sum_{x \in Q} g(x) x
$$

Without loss of generality, for $x \in Q$, either $f(x)>0$ or $g(x)>0$.

We begin by studying absolute reward schedules $(\mathcal{P}, w)$, where $\mathcal{P}$ is the titles partition and $w$ is the wage schedule. Later, in Section 5 , we shall show that relative reward schedules are inferior.

In the absolute schedule, there is a partition $\mathcal{P}$ of $Q$ into consecutive cells (intervals) corresponding to increasing titles; $\mathcal{P}(x)$ denotes the cell of $\mathcal{P}$ in which $x$ lies. There is also a wage schedule given by a weakly monotonic function $w: Q \rightarrow$ $\left[w_{\min }, \infty\right)$, mapping outputs to wages above some stipulated minimum $w_{\min } \geq 0$. If wages are public, then $w$ must be measurable with respect to $\mathcal{P}$, that is constant on each cell of $\mathcal{P}$, meaning that outputs which get the same title cannot get different wages. The collection of wage schedules that are measurable with respect to $\mathcal{P}$ is denoted $\mathcal{W}(\mathcal{P})$. Let $\Pi$ be the (finite) set of partitions of $Q$ into consecutive cells, denoting all possible ways $\mathcal{P}$ of allocating titles. Let $\mathcal{W}$ denote the collection of all wage schedules, i.e. the set of all weakly monotonic functions $w: Q \rightarrow\left[w_{\min }, \infty\right)$.

Let $I(\mathcal{P})$ denote the status incentive generated by $\mathcal{P}$ when $\sigma_{n}=\sigma=1$, i.e. the increase in payoff of an employee when he switches from shirk to work, assuming that

whereas we concentrate on an absolute scale, after proving that in our context absolute is better for the owner than any relative scale. Finally, they reach a striking conclusion that the top rank should always consist of a single employee. This hinges on the assumption that agents are ex ante identical. Were there sharp ex ante heterogeneities, the conclusion would fail, because effort would not enable the weak to improve their rank by equalling the strong. Even in our homegeneous case, the highest cell does not consist of a single output, except under the very special assumption that higher effort leads to uniform stochastic dominance in performance.

${ }^{22}$ If $Q$ is a compact interval, we can approximate it by a fine finite grid and then use a limiting argument to derive the analogous result for a continuum of outputs.

${ }^{23}$ We assume independence for ease of exposition. Our analysis goes through with a weaker hypothesis consisting of two parts. (a) If N-1 employees work and one shirks, then the shirker's performance $\mathrm{g}$ is independent of the workers' performance, each of which is given by $\mathrm{f}$ (which need not be independent from each other). (b) If they all work, their outputs (which can be distributed according to $h \neq f)$ are ex ante symmetric in the following precise sense: consider an elementary event in which every person is assigned an output, and another elementary event obtained by permuting the names; then the two elementary events should have the same probability. 
all others are working, ignoring money altogether and considering only titles. (The status incentive for arbitrary $\sigma \geq 0$ is then $\sigma I(\mathcal{P})$.) Clearly his status payoff is 0 when he works, since he comes ahead of his ex ante identical competitors as often as he comes behind. Therefore, recalling that performances are independent, his status incentive is simply $N-1$ times the negative of his status payoff when he shirks and faces exactly one competitor who works.

$$
I(\mathcal{P})=(N-1)\left[\sum_{\{x \in Q, y \in Q: \mathcal{P}(y)<\mathcal{P}(x)\}} f(x) g(y)-\sum_{\{x \in Q, y \in Q: \mathcal{P}(y)>\mathcal{P}(x)\}} f(x) g(y)\right]
$$

Let $I^{S}$ denote the maximum incentive to work that can be generated by status alone, i.e.

$$
I^{S}=\max _{\mathcal{P} \in \Pi} I(\mathcal{P})
$$

As we shall see in Section 3.1, it follows from our productivity assumption that $I^{S}>0$.

Similarly, given a wage schedule $w \in \mathcal{W}$, we can define the wage incentive

$$
I(w)=\sum_{x \in Q}[f(x)-g(x)] u(w(x))
$$

and the maximum incentive to work that can be created by money alone

$$
I^{M}=\sup _{w \in \mathcal{W}} I(w)
$$

A popular but naive wage schedule is the piece rate in which $w(x)=\lambda x$ for some fixed scalar $\lambda>0$. If $u$ is linear, the piece rate creates a positive incentive to work (on account of the productivity assumption). By increasing $\lambda$, the incentive can be increased to any level desired. ${ }^{24}$

The piece rate is the first wage schedule that comes to mind, because we are so used to competitive markets. If the worker could sell pieces of his ouput to different competing firms, then a market price would be established for his output, corresponding to the piece rate. But this logic does not apply to our setting. The worker can choose among different firms (modeled by his participation constraint, which we introduce at the end of Section 2) but having made the choice he becomes an employee and must give his entire output to the owner. As long as they remain with the firm, the employeess are paid according to the policy set by the owner. As we shall see, the owner will not want to set a piece rate.

\footnotetext{
${ }^{24}$ More generally, even if $u$ is not linear, we can define a wage schedule $\tilde{w}(x)$ so that $u(\tilde{w}(x))$ is linear in $x$. Indeed, fix $0<\lambda<\left(\sup _{w \in \mathbb{R}} u(w)-u\left(w_{\min }\right)\right) /\left(x_{\max }-x_{\min }\right)$. Let $\tilde{w}(x)=u^{-1}\left(u\left(w_{\min }\right)+\right.$ $\left.\lambda\left(x-x_{\min }\right)\right)$ for all $x \in Q$.

From the productivity assumption, this wage schedule gives positive incentive to work. Hence $I^{M}>0$. Furthermore, if $u(w) \rightarrow \infty$ as $w \rightarrow \infty$, then we can take $\lambda$ arbitrarily large and the incentive to work becomes arbitrarily large, hence $I^{M}=\infty$.

The piece rate schedule and its adapted version for concave $u$ is simple but not economical. We shall shortly derive a much less costly wage schedule that gives the same incentive to work.
} 
Consider the general problem of selecting the optimal reward (i.e. wage-title) schedule, taking into account both the status incentives of titles and the consumption incentives of wages. ${ }^{25}$

$$
\begin{aligned}
\min _{w, \mathcal{P}} & \sum_{x \in Q} f(x) w(x) \\
\text { s.t. } & \left\{\begin{array}{l}
\sigma I(\mathcal{P})+I(w) \geq d \\
\mathcal{P} \in \Pi, w \in \mathcal{W} \\
w \in \mathcal{W}(\mathcal{P}) \text { if wages are public }
\end{array}\right.
\end{aligned}
$$

The optimal absolute reward schedule turns out to be quite simple for ex ante identical employees. To find it we break the analysis into two parts. Throughout we keep $f, g$ fixed, and examine the solution as $d$ varies.

In Section 3.1 we solve the pure titles problem. We ask how the owner could best use titles to motivate his employees to work, without handing out any money at all, i.e., we characterize all partitions $\mathcal{P}$ such that $I(\mathcal{P})=I^{S}$. It turns out that these partitions can be identified by the easily checked "inside and outside" conditions. Furthermore, we show that they form a (complete) sublattice of the lattice of all partitions, with maximal element $\mathcal{P}^{*}$ and minimal element $\mathcal{P}_{*}$. It is evident that this sublattice is the set of all solutions to the minimization problem above, for each $d \leq \sigma I^{S}$; and that there are no solutions without wages when $d>\sigma I^{S}$. Finally, for generic $f, g$ the sublattice is a singleton.

A typical property of optimal partitions is that they are coarse, clumping many outputs into the same cell: there are far fewer titles than outputs. In fact, only in the very special scenario where $f$ uniformly stochastically dominates $g$ do we get as many titles as outputs in an optimal solution.

In Section 3.2 we solve the pure wage problem. We ask how the owner should best choose a wage schedule when her employees derive no status utility from titles, i.e., which $w_{d} \in \mathcal{W}$ solve the minimization problem above when $\sigma=0$ ? Solutions exist for all $d \leq I^{M}$. We show that any solution to the pure wage problem is connected to the pure titles problem. Every solution $w_{d}$ of the pure wage problem is measurable with respect to the finest partition $\mathcal{P}^{*}$ that solves the pure titles problem, and there exists a solution that is measurable with respect to the coarsest partition $\mathcal{P}_{*}$.

Thus the optimal wage schedule gives no more wage levels than there are titles, and possibly fewer. This is a far cry from a piece rate schedule where wages strictly increase with each output. The optimal wage schedule is a step function with broad steps. At the jump points, a small increase in output is rewarded with a huge increase in wage.

This is even more starkly true when employees are risk neutral. In this case, $w_{d}$ can be taken to be a "trigger wage" or "star wage": outputs below a threshold $q^{*}$ are paid $w_{\min }$ and those above $q^{*}$ get a bonus $w_{\min }+B$, i.e. we get just two wage levels

\footnotetext{
${ }^{25}$ We implicitly assume that the output of the worker is so valuable to the employer relative to the wages he needs to pay in order to get them to work, that he deems it optimal to incentivize everyone to work.
} 
no matter how many outputs. Moreover, for generic $f$ and $g$, the optimal wage must be of the trigger form. In the special case where $f$ uniformly stochastically dominates $g$, the bonus is given only to the very top element of $Q$ and the trigger wage is really a "star's" wage.

When titles and wages are combined, the optimal wage-title schedule is achieved simply by the superposition of the pure titles solution and the pure wage solution just described, and this is so whether wages are public or secret. ${ }^{26}$ Since the owner is trying to minimize the wage bill, he will first try to see how far he can go via titles alone before putting up money to motivate his workers. We find that for all $0<d \leq \sigma I^{S}$, it is optimal to choose any partition $\mathcal{P}$ that solves the pure titles problem. No wages are necessary. For $\sigma I^{S}<d \leq \sigma I^{S}+I^{M}$, the same titles partition $\mathcal{P}$ can be accompanied by the wage schedule $w_{d-\sigma I^{S}}$ that solves the pure wage problem for disutility $d-\sigma I^{S}$. It makes no difference whether wages are public or secret. For $d>\sigma I^{S}+I^{M}$, no solution is possible.

When $u$ is concave and satisfies increasing relative risk aversion, we show that as $\sigma$ increases the optimal wage schedule becomes more and more trigger like. Thus as society becomes more status conscious, wages become more unequal. We prove these results over the next three sections.

\section{$2.1 \quad$ Titles Alone}

We examine the incentive to work created by titles alone, and ask which $\mathcal{P}$ maximizes $I(\mathcal{P})$. Such a $\mathcal{P}$ is optimal in the sense that if any other title schedule $\mathcal{P}^{\prime} \in \Pi$ gets employees to work via status incentive alone, so will $\mathcal{P}$. To characterize optimal $\mathcal{P}$, we need to recall two notions of stochastic dominance.

\subsubsection{Stochastic Dominance}

Definition: Let $X$ and $Y$ be independent random variables which take on values in a finite totally ordered set $Z \cdot{ }^{27}$ We say that $X$ (stochastically) dominates $Y$ on the interval $[a, b] \subset Z$ if $\operatorname{Pr}(X \in[a, b]) \operatorname{Pr}(Y \in[a, b])=0$, or

$$
\operatorname{Pr}(X \in[\theta, b] \mid X \in[a, b])-\operatorname{Pr}(Y \in[\theta, b] \mid Y \in[a, b]) \geq 0,
$$

for all $\theta \in(a, b]$. In this case we write

$$
X \succsim Y \text { on }[a, b]
$$

In words, this means that no matter at what point $\theta$ we cut the interval $[a, b]$, conditional on both $X$ and $Y$ lying in $[a, b], X$ is at least as likely to lie in the upper segment as $Y$. If every cut on $[a, b]$ gives a strict inequality, then we say that $X$ strictly dominates $Y$ and we denote it by $X \succ Y$ on $[a, b]$.

\footnotetext{
${ }^{26}$ This is surprising because the pure titles partition implements work as a Nash equilibrium in the $\mathrm{N}$-person game, whereas the optimal pure wage schedule implements work in a one-person problem.

${ }^{27}$ As before, we assume without loss of generality that for each $a \in Z$, either $\operatorname{Pr}(X=a)>0$ or $\operatorname{Pr}(Y=a)>0$.
} 
With this definition in hand, we can show that $I^{S}>0$. Let $X \sim f$ and $Y \sim g$ denote the stochastic outputs of the worker and the shirker. First note that $Y$ cannot stochastically dominate $X$ on $Q$, otherwise, by the "dominance increases expectation lemma" in the appendix, $\sum_{x \in Q} f(x) x \leq \sum_{x \in Q} g(x) x$, contradicting the productivity assumption. Therefore there exists a $\theta \in Q$ such that $\operatorname{Pr}(X \geq \theta)-\operatorname{Pr}(Y \geq \theta)>0$. Partition $Q$ into two titles: let all outputs less than $\theta$ be accorded the low title, and all outputs $\theta$ and above be given the high title. This clearly generates positive status incentive, hence $I^{S}>0$.

A moment's thought will convince the reader that $X \succsim Y$ on $[a, b]$ if and only if whenever $[a, b]=L \cup R$ is divided into two disjoint intervals, the left interval $L$ lying below the right interval $R$, then

$$
\frac{P(X \in L)}{P(Y \in L)} \leq \frac{P(X \in R)}{P(Y \in R)}
$$

This is obviously equivalent to the "betweenness" property

$$
\frac{P(X \in L)}{P(Y \in L)} \leq \frac{P(X \in L \cup R)}{P(Y \in L \cup R)} \leq \frac{P(X \in R)}{P(Y \in R)}
$$

In the case of strict domination, these inequalities will be strict.

It will be useful to consider a strengthened form of domination.

Definition: We say that $X$ uniformly dominates $Y$ on the interval $[c, d] \subset Z$ if $X$ dominates $Y$ on every subinterval $[a, b] \subset[c, d]$. In this case we write $X \succsim_{U} Y$ on $[c, d]$.

Uniform domination $X \succsim_{U} Y$ on $[c, d]$ can easily be seen to be equivalent to the condition that whenever $a<b$ are consecutive elements of $[c, d]$ then

$$
\frac{P(X=a)}{P(Y=a)} \leq \frac{P(X=b)}{P(Y=b)}
$$

In case $[c, d]$ consists of two elements, domination and uniform domination are the same. But with three elements or more, uniform domination is a strictly stronger requirement. Strict uniform domination, denoted $X \succ_{U} Y$, is defined just like uniform domination, but with strict inequalities throughout.

\subsubsection{The Optimal Titles Partition}

To create the best incentives for work, we need to lower the shirker's payoff as much as possible. Thus it stands to reason that we should mask performance in regions of ouput where the shirker is better than the worker, by awarding the same title throughout; and award titles for superior performance across regions where the worker is likely to do better. These are reflected in the inside and outside domination conditions of our first Theorem. 
Theorem 1 (Inside-Outside Condition): Let $X, Y$ denote the random output of the employee when he works, shirks. Then $I(\mathcal{P})$ is maximized over $\Pi$ at $\bar{P}$ if and only if

(i) Inside Domination: $Y \succeq X$ on each cell of $\bar{P}$

(ii) Outside Uniform Domination: $X \succeq_{U} Y$ across the cells of the ordered set $\bar{P}$

(The proof of Theorem 1 and all other omitted proofs can be found in the Appendix.)

Given that $X$ and $Y$ both lie somewhere in two consecutive partition cells, condition (ii) says that $X$ is more likely than $Y$ to lie in the upper cell. But given that $X$ and $Y$ lie in the same cell, condition (i) says that it is more likely that $Y$ is to the right of any cut.

One might have thought that since titles create status incentive and are free to bestow, the owner should hand out as many titles as he can. However, an optimal partition often involves masked cells. Indeed we have

Lemma 1 (Coarse Partition): Suppose that $x<y$ are consecutive outputs and that

$$
\frac{f(x)}{g(x)}>\frac{f(y)}{g(y)}
$$

Then $x$ and $y$ must be in the same cell of any optimal titles partition. In particular, an optimal titles partition can be perfectly fine (and hand out as many titles as there are outputs) only if $X \succeq_{U} Y$.

In the special case where the worker uniformly dominates the shirker, we do get the opposite.

Lemma 2 (Fine Partition): Suppose $X \succeq_{U} Y$. Then the perfectly fine partition is optimal. If $X \succ_{U} Y$, then the perfectly fine partition is the unique optimum.

For examples and discussion see Dubey-Geanakoplos (2005, 2010).

The set of all optimal partitions turns out to be a lattice. Recall that the join of two partitions is the coarsest partition that refines them both, and that the meet is the finest partition that they both refine. In our case of interval partitions of totally ordered finite sets, the partitions are easily identified with their cuts, i.e. the boundary points of the intervals. ${ }^{28}$ Then the join of two partitions is defined by the union of their cuts, and the meet is defined by the intersection of the cuts.

\footnotetext{
${ }^{28}$ Formally speaking, a cut is defined by a pair $a b$ where $a$ is a last element of one interval and $b$ is the first element of the next interval.
} 
Theorem 2 (Lattice Structure): The optimal partitions form a sublattice, under the join and meet operations, of the lattice of all partitions. Thus there is a unique optimal title partition $\mathcal{P}^{*}$ with the most titles, obtained by taking the join of all the optimal partitions; this maximal optimal partition is the unique optimal partition that displays strict inside domination on each of its cells. There is also a unique optimal title partition $\mathcal{P}_{*}$ with the fewest titles, obtained by taking the meet of all the optimal partitions; this minimal optimal partition is the unique optimal partition that displays strict outside domination. Finally, the sublattice is complete, i.e. it includes all the elements of the lattice between the meet and the join. Indeed, each cell in any partition in the sublattice is the union of consecutive cells $C$ from the maximal optimal partition across which $f(C) / g(C)$ is constant.

According to Theorem 2, every optimal partition is obtained by consolidating some of the titles of the maximal optimal partition or equivalently by splitting some of the titles of the minimal optimal partition. For example, the fewest titles (in the minimal optimal title partition) might be general, colonel, major, captain, lieutenant, sargeant, corporal, private. The most titles (in the maximal optimal title partition) might be lieutetant general, major general, brigadier general, ..., private first class, private second class and so on.

The proof of Theorem 1 was given in a more general setting in Dubey-Geanakoplos $(2005,2010)$. For completeness, and because the proof is so much simpler and possibly more instructive in the finite output case considered in this paper, we present it in the Appendix. Theorem 2 is presented here for the first time.

It is worth noting that the lattice is usually a singleton.

Lemma 3 (Unicity of the Lattice): Regarding $f$ and $g$ as vectors in the finite dimensional set $\mathbb{R}^{Q}$, the optimal partiton is unique for (Lebesgue) almost all $f, g$.

\subsubsection{Computing the Minimal and Maximal Optimal Titles Partitions}

We shall provide two algorithms for computing the minimal optimal partition $\mathcal{P}_{*}$. Once we have $\mathcal{P}_{*}$ it is straightforward to construct $\mathcal{P}^{*}$.

The First Algorithm for the Minimal Optimal Partition Start with the finest possible partition of $Q$ into singleton cells. Trivially this partition satisfies the inside condition. Proceed inductively as follows.

Given any partition $(\ldots D<C<B<A)$ satisfying the inside condition on each cell, starting from the right look at all pairs of consecutive cells, $B A, C B, D C$, etc. Take the first pair $\beta \alpha$ in the list for which

$$
\frac{f(\beta)}{g(\beta)} \geq \frac{f(\alpha)}{g(\alpha)}
$$

If no such pair can be found, then by the inside-outside condition of Theorem 1 , we have an optimal partition; and, by the lattice structure of Theorem 2, it is the minimal optimal partition. 
Otherwise, combine cells $\beta$ and $\alpha$ into the bigger cell $\beta \cup \alpha$. By the merger lemma in the Appendix, this new partiton must also satisfy the inside condition on each of its cells. Iterate the process. Since $Q$ is finite, the process must terminate.

\section{The Second Algorithm for the Minimal Optimal Partition Let}

$$
\theta_{1}^{*}=\operatorname{argmax}_{\theta \in Q} \frac{\sum_{x \geq \theta} f(x)}{\sum_{x \geq \theta} g(x)}
$$

If there are multiple such maximizers, choose the smallest. Then define the rightmost cell as $C_{1}=\left\{q \in Q: \theta_{1}^{*} \leq q\right\}$. $^{29}$

Given $\theta_{k}$, define

$$
\theta_{k+1}^{*}=\operatorname{argmax}_{\theta<\theta_{k}} \frac{\sum_{\theta_{k}>x \geq \theta} f(x)}{\sum_{\theta_{k}>x \geq \theta} g(x)}
$$

Again choose the lowest such maximizers in case there are ties. Then define $C_{k+1}=$ $\left\{q \in Q: \theta_{k+1}^{*} \leq q<\theta_{k}^{*}\right\}$. This algorithm terminates in a partition after at most $|Q|$ steps.

It remains to check that the constructed partition satisfies the inside and outside conditions. But this is evident. If

$$
\frac{f\left(C_{k+1}\right)}{g\left(C_{k+1}\right)} \geq \frac{f\left(C_{k}\right)}{g\left(C_{k}\right)}
$$

then by betweenness, $\theta_{k+1}$ would have done at least as well as $\theta_{k}$ in the kth maximization problem, and been lower, a contradiction. This establishes strict outside domination.

For any cut of $C_{k}$ into $\left[\theta_{k}, \theta\right) \cup\left[\theta, \theta_{k-1}\right)$ we must have

$$
\frac{f\left(\left[\theta_{k}, \theta\right)\right)}{g\left(\left[\theta_{k}, \theta\right)\right)} \geq \frac{f\left(\left[\theta, \theta_{k-1}\right)\right)}{g\left(\left[\theta, \theta_{k-1}\right)\right)}
$$

otherwise, by the betweenness property, $\theta$ would do better than $\theta_{k}$ in the $k$ th maximization problem. This establishes inside domination.

Theorems 1 and 2 imply that we have found $\mathcal{P}_{*}$.

Algorithm for the Maximal Optimal Partition Given any optimal partition $\mathcal{P}$, it is a simple matter to construct the maximal optimal partition $\mathcal{P}^{*}$. Simply look at all cuts of any cell $C$ in $\mathcal{P}$. If the cut leaves $f / g$ the same on both sides, make it. By the splitting lemma in the appendix, the new partition satisfies the inside-outside conditions. Continue iterating the process. By Theorem 2 (Lattice Structure) the algorithm can only stop at the maximal optimal partition.

\footnotetext{
${ }^{29}$ For this algorithm we depart from the convention we use elsewhere, and number the topmost cell 1 and the bottom cell $\mathrm{K}$, instead of vice versa.
} 


\subsection{Wages Alone}

Now we turn to the classical pure wage problem where employees do not care about status, but only about money, and hence must be motivated by wages alone. Surprisingly, we find that no optimal pure wage schedule ever pays differently to outputs that are accorded the same title in the pure titles solution $\mathcal{P}^{*}$. In the pure wage problem people don't compare themselves with each other, and only think about the money they get. In the pure titles problem they don't care about money, but only about how they rank against others. Nonetheless, the solutions to these diametrically opposed problems are in harmony. Indeed, our characterization of the optimal titles partition vastly simplifies the search for the optimal pure wage schedule.

From now on we shall make the not unrealistic assumption that the disutility of work is high enough that no employee will work for status alone, by requiring

The Necessity-of-Wages Assumption (Homogeneous Case):

$$
d>\sigma I^{S}
$$

Theorem 3 (Compatibility of Pure Wages and Pure Titles): Let $P(w)$ be the partition induced ${ }^{30}$ by any solution $w$ of the pure wage problem

$$
\begin{aligned}
& \min _{w \in \mathcal{W}} \sum_{x \in Q} f(x) w(x) \\
& \text { s.t. } I(w) \equiv \sum_{x \in Q}[f(x)-g(x)] u(w(x)) \geq d
\end{aligned}
$$

Then $\mathcal{P}(w) \subset \mathcal{P}^{*}$. Furthermore, there exists a solution $w$ such that $\mathcal{P}(w)=\mathcal{P}_{*}$.

The intuition for Theorem 3 is that we can always replace the wage schedule inside a cell of $\mathcal{P}^{*}$ with a constant wage chosen so as to leave unchanged the expected utility of the worker inside the cell. From the worker's risk aversion we deduce that this does not increase the expected wage payment of the owner. Since the shirker strictly dominates the worker inside each cell, this maneuver strictly hurts the shirker. Thus, lowering the constant wage inside the cell just a tiny bit more improves the profits of the owner and still increases the incentive to work.

The next theorem shows that every optimal pure wage schedule pays the minimal wage for a nontrivial initial segment of outputs.

Theorem 4 (Minimum Wage) Let the maximal optimal titles partition $\mathcal{P}^{*}$ consist of consecutive cells $C_{1}<\ldots<C_{K}$. Let $f\left(C_{k}\right) \leq g\left(C_{k}\right)$. (Clearly there must be one such $k$, since $\left.\sum f\left(C_{k}\right)=1=\sum g\left(C_{k}\right)\right)$. Then, for every solution $w$ of the pure wage problem given above, $w(x)=w_{\min }$ for all $x \in C_{1} \cup \ldots \cup C_{k}$.

\footnotetext{
${ }^{30}$ Any function induces a partition of its domain consisting of sets on which it has a constant value. If the function is weakly monotonic, as our wages are, this partition consists of consecutive cells.
} 
The intuition for Theorem 4 is as follows. From the outside condition that $f\left(C_{i}\right) / g\left(C_{i}\right)$ is increasing in $i$, we know that $f\left(C_{i}\right) \leq g\left(C_{i}\right)$ for all $i \leq k$. Lowering the wage on all these cells to $w_{\text {min }}$ reduces outlays for the owner and cannot hurt the incentive to work.

A surprising Corollary of Theorem 4 is that with strict risk aversion, there is a unique optimal pure wage schedule.

Corollary to Theorem 4 (Uniqueness with Strict Risk Aversion) If $u$ is strictly concave, then the pure wage problem has a unique solution.

The reason is simple. If there were two optimal wage schedules, $w$ and $w^{\prime}$, then their average $w^{*}$ would be monotonic and would cost the owner the same. By Theorem $4, w^{*}$ would be the same as $w, w^{\prime}$ for every cell with $f\left(C_{i}\right) \leq g\left(C_{i}\right)$. But for all other cells, $\left[f\left(C_{i}\right)-g\left(C_{i}\right)\right] u\left(w_{i}^{*}\right)>\left[f\left(C_{i}\right)-g\left(C_{i}\right)\right]\left(.5 u\left(w_{i}\right)+.5 u\left(w_{i}^{\prime}\right)\right)$ whenever $w_{i} \neq w_{i}^{\prime}$, showing that the incentive to work with $w^{*}$ is strictly higher than the incentive to work of $w, w^{\prime}$. Thus we can lower the wages in $w^{*}$ by a tiny bit and make the owner strictly better off.

The next theorem shows that there is always an optimal pure wage schedule that is measurable with respect to $\mathcal{P}_{*}$ and pays as before the minimum wage for an initial segment of cells, but is strictly increasing across all cells thereafter.

Theorem 5 (Wage Structure) Let $w$ be an optimal wage schedule and let its induced partition $P(w)$ consist of consecutive cells $C_{1}<\ldots<C_{L}$, with associated wages $w_{\min }=w_{1}<\ldots<w_{L}$. Let employee utility $u$ be differentiable. Then for $\ell>1$ we have

$$
\frac{u^{\prime}\left(w_{\ell+1}\right)}{u^{\prime}\left(w_{\ell}\right)}=\frac{\left[f\left(C_{\ell}\right)-g\left(C_{\ell}\right)\right] / f\left(C_{\ell}\right)}{\left[f\left(C_{\ell+1}\right)-g\left(C_{\ell+1}\right)\right] / f\left(C_{\ell+1}\right)}
$$

while for $\ell=1$ the $=$ must be replaced by $\geq$.

The proof of these formulae is as follows. If $w_{\ell+1}>w_{\ell}>w_{\min }$, then we can consider transferring an expected, infinitesimal, dollar from cell $C_{\ell+1}$ to $C_{\ell}$, or vice versa. This is tantamount to increasing (decreasing) $w_{\ell+1}$ by $1 / f\left(C_{\ell+1}\right)$ dollars and decreasing (increasing) $w_{\ell}$ by $1 / f\left(C_{\ell}\right)$ dollars. The incentive effect is the difference between $u^{\prime}\left(w_{\ell+1}\right)\left[f\left(C_{\ell+1}\right)-g\left(C_{\ell+1}\right)\right] / f\left(C_{\ell+1}\right)$ and $u^{\prime}\left(w_{\ell}\right)\left[f\left(C_{\ell}\right)-g\left(C_{\ell}\right)\right] / f\left(C_{\ell}\right)$. These are equal if we are at an optimum, explaining the equality. If $w_{\ell}=w_{\min }$ then the transfer can only go one way, and we get the inequality. 5 .

In fact by the same argument it is possible to prove a stronger version of Theorem

Theorem $5^{\prime}$ (Wage Structure) Let $w$ be an optimal wage schedule and let the maximal partition $\mathcal{P}^{*}$ consist of consecutive cells $C_{1}<\ldots<C_{L}$, with associated wages $w_{\min }=w_{1} \leq \ldots \leq w_{L}$. Let employee utility $u$ be differentiable. Then if $w_{\ell}>w_{\min }$, we have

$$
\frac{u^{\prime}\left(w_{\ell+1}\right)}{u^{\prime}\left(w_{\ell}\right)}=\frac{\left[f\left(C_{\ell}\right)-g\left(C_{\ell}\right)\right] / f\left(C_{\ell}\right)}{\left[f\left(C_{\ell+1}\right)-g\left(C_{\ell+1}\right)\right] / f\left(C_{\ell+1}\right)} ;
$$


otherwise $=$ must be replaced by $\geq$.

If the two cells are part of a big slab of constant wages, then extend the bottom cell to the left end of the slab and extend the top cell to the right end of the slab. Now the owner can profitably adjust the wages across the two parts of the slab if the equality fails. (Since $f / g$ is increasing across cells of $\mathcal{P}^{*}$, an inequality will induce an increase in the right part of the slab and a decrease in the left, which does not violate monotonicity.)

The right hand side is less than 1 whenever $f\left(C_{\ell+1}\right) / g\left(C_{\ell+1}\right)>f\left(C_{\ell}\right) / g\left(C_{\ell}\right)$. Theorem $5^{\prime}$, together with our observation from Theorem 3 that there exists an optimal wage schedule that is measurable with respect to $P_{*}$, gives

Corollary to Theorem $5^{\prime}$ (Wage Structure) There is an optimal pure wage schedule $w$ that is measurable with respect to $P_{*}$, such that $w(x)=w_{\min }$ on a nonempty initial segment $x \in C_{1} \cup \ldots \cup C_{\ell^{*}}$ of $P_{*}$, and strictly increasing across those cells to the right of $C_{\ell^{*}}$.

In the special case when employees are risk neutral towards money (i.e. $u$ is linear), the equality of Theorem $5^{\prime}$ cannot hold if $f / g$ is strictly increasing from $C_{\ell}$ to $C_{\ell+1}$. Hence $w=w_{\min }$ all the way up to the top cell of $\mathcal{P}_{*}$, after which $f / g$ is constant across cells of $\mathcal{P}^{*}$. Thus there is an optimal trigger wage, which is $w_{\min }$ on every cell in $\mathcal{P}_{*}$ below the top cell and $w_{\text {min }}$ plus a positive bonus $B$ for all outputs in the top cell. (The lowest element $\theta$ of the top cell triggers the bonus). This is the content of Theorem 6 below.

Theorem 6 (Trigger Wage with Risk Neutrality): If $u$ is linear, then every optimal wage schedule must assume the constant value $w_{\min }$ for all outputs below the top cell of $\mathcal{P}_{*}$. For any optimal titles partition $\mathcal{P}$, there exists an optimal pure wage schedule $w$ of the trigger form that pays $w_{\text {min }}$ for all outputs below the top cell of $\mathcal{P}$ and a bonus $w_{\min }+B$ to outputs in the top cell of $\mathcal{P}$, where

$$
B=\frac{d}{\sum_{x \geq \theta}[f(x)-g(x)]}
$$

and $\theta$ is the lowest output in the top cell of $\mathcal{P}$. For almost all $f$ and $g$, this is the unique optimal wage schedule.

Park (1995) derived a trigger wage for risk neutral workers under the much stronger hypothesis that $f$ uniformly dominates $g$. He also did not consider risk averse workers. (He did, however, allow for multiple levels of effort, which under under uniform domination, can be accomodated in our model as well; see the Remark after Theorem 10). Besides being more general, our approach is also different from Park's, in that we link the pure wage problem to the pure titles problem whereas he does not have titles at all.

In the next theorem we ask what happens to the optimal wage schedule when the disutility of work falls from $e$ to $d$. We shall show first of all, that wages can be 
lowered, i.e. $w_{d}(x) \leq w_{e}(x)$ for all $x$, but once the optimal wage $w_{d}$ rises above $w_{\min }$, both jump at exactly the same outputs.

Lemma 4 (Disutility and Wage Jump Points with Strict Risk Aversion) Let employee utility $u$ be differentiable and strictly concave. Suppose $w_{d}, w_{e}$ are solutions of the pure wage problem above for disutilities $d<e$. Then $w_{d}(x)<w_{e}(x)$ whenever $w_{e}(x)>w_{\min }$ and $w_{d}(x)=w_{e}(x)$ whenever $w_{e}(x)=w_{\min }$. If $w_{d}(x)>w_{\min }$, and $y>x$, then $w_{d}(y)>w_{d}(x)$ if and only if $w_{e}(y)>w_{e}(x)$.

From Theorem 3, we can take the optimal wage schedules to be measurable with respect to $P_{*}$, (and by the Corollary to Theorem 4 these are the only optimal wage schedules). By Theorem $5^{\prime}$, each wage schedules jumps at every cut point of $P_{*}$ once it is past $w_{\min }$.

With risk averse employees, we might see a gradual increase in wages as we go up the productivity ladder. The next theorem shows that as the disutility of effort falls, the optimal pure wage schedule becomes more trigger like, as long as $u$ displays increasing relative risk aversion. By more trigger like, we mean that the percentage increase in wages is always higher for $w_{d}$ than for $w_{e}$.

Theorem 7 (Trigger-Like Wages with Strict Risk Aversion): Let $d<e$. Let employee utility $u$ be twice differentiable and strictly concave. Suppose in addition that $u$ displays increasing relative risk aversion, i.e. suppose

$$
-\frac{u^{\prime \prime}(x)}{u^{\prime}(x)} x
$$

is weakly increasing in $x$. Let $w_{\min } \geq 0$. Then $w_{d}$ looks more like a trigger wage schedule than $w_{e}$ in the sense that $x<y$ and $w_{d}(x)>w_{\min }$ implies

$$
\frac{w_{d}(y)}{w_{d}(x)} \geq \frac{w_{e}(y)}{w_{e}(x)}
$$

with strict inequality if $w_{d}(y)>w_{d}(x)$ and $u$ displays strictly increasing relative risk aversion.

Increasing relative risk aversion implies that the percentage change in marginal utility for a one percent change in $x$ is rising in $x$. The proof of Theorem 7 follows immediately from Lemma 4 and the equality of Theorem 5.

\subsection{Titles and Wages Together}

Having considered titles and wages separately, we are ready to put them together. One surprise is that the optimal pure titles schedule need not change when wages are added. Imagine a pre-monetary society in which workers were motivated by titles alone. Suppose their disutility of work goes up, requiring further motivation from monetary wages. Then the optimal wage-title deployment would not alter the titles whatsoever, but on the contrary, simply reinforce them by paying wages according to the old titles. 
Theorem 8 (Optimal Wage-Title Schedule): Let wages be secret or public. Let $\sigma I^{S}<d \leq \sigma I^{S}+I^{M}$. (If $d \leq \sigma I^{S}$, wages are unnecessary for motivation and everybody could be paid $\left.w_{\min }\right)$. Suppose $(\mathcal{P}, w)$ is an optimal wage-title schedule. Then $\mathcal{P}$ is a solution for the pure titles problem and $w$ is a solution for the pure wage problem with $d^{*}=d-\sigma I(\mathcal{P})$ in place of $d$. Moreover, for almost all $f$ and $g$, the partition $\mathcal{P}$ is uniquely determined.

Thus with homogenous employees, there is nothing to be gained by keeping wages secret. Moreover, we should in general expect to see far fewer titles given than there are outputs, and far fewer wages than there are titles.

These features come starkly to light when employees are risk neutral. There is only one wage above the minimum, given as a bonus to an elite of top performers. Many title distinctions may occur below the elite, but all of them are paid the minimum wage.

Theorem 9 (Star Wages): If $u$ is linear, then one optimal wage-title schedule $w$ is the trigger wage

$$
w_{(\theta, B)}(x)= \begin{cases}w_{\min } & \text { if } x<\theta \\ w_{\min }+B & \text { if } x \geq \theta\end{cases}
$$

where $\theta$ is the smallest element of the top cell of $\mathcal{P}_{*}$ and

$$
B=\frac{d^{*}}{\sum_{x \geq \theta}[f(x)-g(x)]}
$$

where $d^{*}=d-\sigma I\left(\mathcal{P}_{*}\right)$. For generic $f, g$ this is the unique optimal wage-title schedule.

If $f$ uniformally stochastically dominates $g$, then the unique optimal wage-title schedule gives a different title to every output and pays the minimum wage to every worker who does not achieve the top-most output, and a giant bonus to those who do.

We are now in a position to examine what happens when $\sigma$ rises and society becomes more status conscious. Our main result is that in the presence of increasing relative risk aversion, increasing status has the effect of making the optimal wage schedule more star-like.

Theorem 10 (Status Creates Star-Like Wages): Suppose $u$ is twice differentiable and strictly concave, and displays strictly increasing relative risk aversion. Then as $\sigma$ rises and the agents become more status conscious, wages fall and become more trigger like in the sense (of Theorem 7) that wages always rise by a higher percentage once they exceed $w_{\min }$.

Indeed, the move from zero status to $\sigma$ has the same effect as lowering the disutility by $\sigma I^{S}$ and finding an optimal pure wage schedule for the diminished disutility. 
Remark (Multiple Effort Levels) Suppose utilities are linear and agents $h$ have multiple effort levels $e_{1}^{h}, \ldots, e_{m-1}^{h}, e_{m}^{h}$ with corresponding stochastic outputs $X_{1}, \ldots, X_{m-1}, X_{m}$ (each with full support on the underlying finite space of outputs) and disutilities $d_{i}^{h}$ to switch from effort $e_{i}^{h}$ to maximal effort $e_{m}^{h}$, for $i=1, \ldots, m-1$. Further suppose that $X_{m}$ uniformly stochastically dominates $X_{i}$ for $i=1, \ldots, m-1$. Then, by Theorem 9 , the optimal wage-title schedule that motivates all the agents to put in maximal effort is the perfectly fine partition with a wage schedule that pays the minimum wage everywhere except for a bonus $B$ at the top-most output.

Remark (Participation Constraints) Suppose utilities are concave, not necessarily linear. We could add an ex ante Participation Constraint (PC), over and above the minimal wage requirement, i.e., the (expected) utility any agent gets, from wages and titles combined, should never sink below some stipulated floor $u_{*}$. We can still prove that every optimal wage schedule must be measurable with respect to $P^{*}$. To see this, first note that, when they all work, their (expected) status utility is zero (by symmetry) and thus the utility they enjoy is just their wage utility. Take any wage schedule $w$ that satisfies the incentive constraint and the participation constraint. Suppose $w$ is not measurable with respect to the maximal optimal titles partition $P^{*}$. Then consider $w^{*}$, obtained from from $w$, exactly as in the proof of Theorem 3 . By construction, $w^{*}$ leaves the worker's wage utility unchanged, hence $w^{*}$ satisfies the participation constraint. The shirker's utility is worsened in $w^{*}$ compared to $w$, hence the incentive constraint is satisfied in a stronger manner in $w^{*}$. Finally, the total wage bill is not increased. Thus an optimal wage schedule must be measurable with respect to $P^{*}$.

\section{Disparate Employee Types}

We now turn to the other extreme in which employees have disparate abilities. For the sake of a more succinct presentation, output distributions are taken to be continuous. (The case of discrete outputs is completely analogous, but the formulae become messier).

We imagine disparate employee types $i=1, \ldots, \ell$ arranged in order of ascending abilities, with $N_{i}$ employees of type $i$. As in the homogeneous case, their utility for wage $w$ is given by a common function $u(w)$ and status utility enters as a separable, additive term. But their abilities are no longer taken to be the same. Assuming all the others work, an employee of type $i$ produces output continuously distributed on the high interval $J_{H}^{i}=\left[a_{H}^{i}, b_{H}^{i}\right]$ with density $f_{i}$ when he works, and on the low interval $J_{L}^{i}=\left[a_{L}^{i}, b_{L}^{i}\right]$ with density $g_{i}$ when he shirks. Conditional on others' working, his output depends only on chance and on his own effort, and is independent of all the others' outputs. We assume that abilities are disparate: $J_{L}^{i}<J_{H}^{i}<J_{L}^{i+1}<J_{H}^{i+1}$, i.e., an employee of type $i+1$ is so much more able than an employee of type $i$, that he always comes out ahead even when he shirks and the other works. In particular, the supports of the densities $g_{i}, f_{i}, g_{i+1}, f_{i+1}$ are all disjoint. This corresponds to a situation in which the employees can be clumped into distinct groups with widely 


\section{The Performance Map with Three Types}

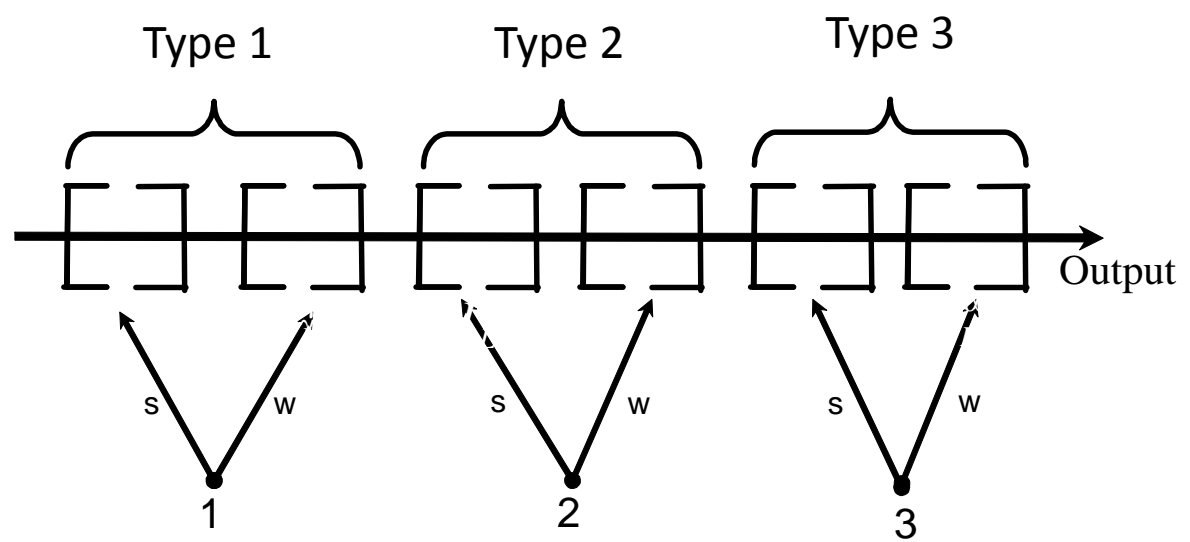

Figure I

different training or experience or expertise. We also suppose that the disutility $d_{i}>0$ for switching from shirk to work can vary across type $i$. (The case when $\ell=1$ is a special instance of the homogeneous employees we discussed in the last Section.) Figure I depicts the case $\ell=3$.

Let $w$ denote a wage function and $\mathcal{P}$ a title partition of the output space. The optimal absolute wage-title schedule $(w, \mathcal{P})$ solves

$$
\begin{aligned}
\min _{w, \mathcal{P}} & \sum_{1 \leq i \leq l} \int f_{i}(x) w(x) d x \\
\text { s.t. } & \left\{\begin{array}{l}
\sigma I^{i}(\mathcal{P})+I^{i}(w) \geq d_{i} \text { for } 1 \leq i \leq l \\
\mathcal{P} \in \Pi, w \in \mathcal{W} \\
w \in \mathcal{W}(\mathcal{P}) \text { if wages are public }
\end{array}\right.
\end{aligned}
$$

where $\Pi$ is the collection of all partitions of the output space $\left[a_{L}^{1}, b_{H}^{l}\right]$ into finitely many consecutive cells; $\mathcal{W}$ is the set of weakly monotonic wage functions on the output space; $\mathcal{W}(\mathcal{P})$ is the set of $w \in \mathcal{W}$ that are measurable with respect to $\mathcal{P}$; the term $I^{i}(w)=\int\left[f_{i}(x)-g_{i}(x)\right] u(w(x)) d x$ is the wage incentive of $i$ under $w$; and, finally, $I^{i}(\mathcal{P})$ is the status incentive of $i$ under $\mathcal{P}$ when all his rivals are working, i.e., the change in his status payoff when he unilaterally switches from shirk to work (we refer forward to the proof of Theorem 12 in the Appendix, for the precise formulae for $I^{i}(\mathcal{P})$, for partitions $\mathcal{P}$ that matter in the optimization).

As in the previous Section with homogeneous employees, it will be useful to examine first the optimal pure title schedule when wages cannot be paid, and then 
the optimal pure wage schedule when titles cannot be conferred. We pass over these two cases quickly to get to the interesting interplay between wages and titles that was absent in the homogeneous case.

We find that the optimal titles partition gives as many titles as there are employee types, far fewer than the continuum of output levels. Typically the optimal pure title partition does not give the highest title to all the employees of the highest type, and allows for the best performers of the lowest type (and in fact all types below the top type) to gain a title equal to the worst performers among those one ability rank higher. In contrast, the optimal pure wage schedule has a simple structure: it always gives the same wage to every worker of type $i-1$ along with every shirker of type $i$; with the jump in wage from the $i$-shirker to the $i$-worker just enough to compensate $i$ for his disutility of effort.

In sharp contrast to the homogeneous case, we find that when titles and wages are allocated together, the optimal titles partition changes; and, moreover, secret and public wages make for quite different solutions. We shall show that when wages are public, the top ability types should be motivated almost entirely by wages, and hardly at all by status (though they get the highest status). A tiny group of elite performers among the top ability type should get astronomical wages. As status becomes more important, the disparity in pay between the highest types and all the other types increases; in short, wages become more star-like. On the other hand, we show that when wages are secret, there should be only two titles, so that all the status incentive is concentrated on the lowest ability type, and everyone else is motivated by wages.

Until the very end of this section we shall assume that $\sigma=1$. As we said earlier, this is without loss of generality, since it can always be achieved be appropriately rescaling utilities.

\subsection{Titles Alone}

Once again titles will be given on the basis of performance as measured by a partition $\mathcal{P}$ of the output space into consecutive cells, as in the last section with homogeneous workers. Assuming all others are working, the expected status payoff to an employee when he works/shirks is given by the expected number of people he beats (according to $\mathcal{P}$ ) minus the expected number of people who beat him. His status incentive to work, $I^{i}(\mathcal{P})$, is his expected status payoff when he works minus his expected status payoff when he shirks.

Theorem 11 (Optimal Pure Titles): Suppose there are $\ell$ disparate types, and a title partition that gives positive status incentive to work for every employee. Then there is another title partition consisting of $\ell$ cuts $c_{1}<\ldots<c_{\ell}$, with $c_{1}=a_{H}^{1}$, and exactly one cut $c_{i}$ in every $J_{H}^{i}, i \geq 2$, which improves (or leaves unchanged) the status incentive to work of every employee.

In view of Theorem 11, we may restrict attention to partitions $\mathcal{P}(p)$ defined

by vectors $p=\left(p_{1}, p_{2}, \ldots, p_{l}\right)$ with $p_{1}=1$. Here $p_{i}=\int_{c_{i}}^{b_{i}^{H}} f_{i}(x) d x$ denotes the 


\section{The Optimal Pure Titles Partition $\boldsymbol{P}\left(p_{1}, p_{2}, p_{3}\right)$}

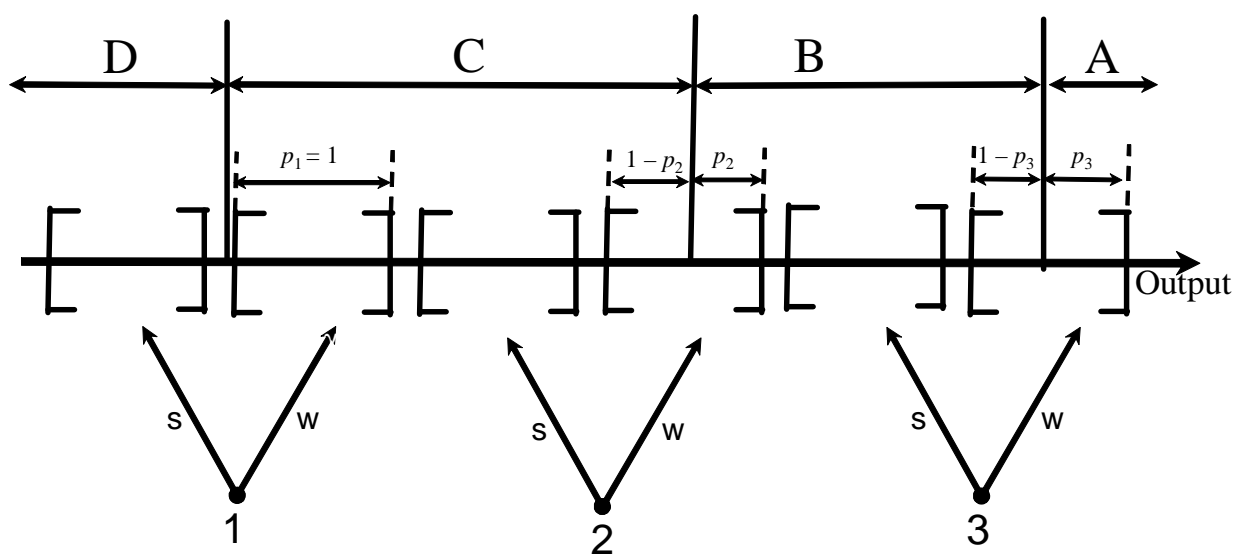

Figure II

probability of the upper tail $J_{H}^{i}\left(c_{i}\right)=\left\{q \in J_{H}^{i}: q \geq c_{i}\right\}$. Since the density $f_{i}$ may be zero on some intervals, there may be several $c_{i}$ that give upper tails with the same probability. In this case it is harmless to choose the lowest such cut $c_{i}\left(p_{i}\right)=\min \{q \in$ $\left.J_{H}^{i}: \int_{q}^{b_{i}^{H}} f_{i}(x) d x=p_{i}\right\}$. (See Figure II below for a partition with three such cuts.)

Denote by $I^{i}(p)$ the status incentive of $i$ that is generated by $\mathcal{P}(p) .{ }^{31}$ In the case where all disincentives $d_{i}=d$, it is natural to maximize the minimum incentive to work, i.e. to choose $p \in[0,1]^{\ell}$ to solve

$$
\max _{p \in[0,1]^{\ell}} \min _{1 \leq i \leq \ell} I^{i}(p)
$$

since this will incentivize everyone to work for the highest possible value of $d$.

Corollary to Theorem 11 Consider the setting of Theorem 11, with $2 \leq N_{1} \leq$ $N_{2} \leq \ldots \leq N_{\ell}$. Then there is a unique $p$ which achieves $\max _{q \in[0,1]^{l}} \min _{1 \leq i \leq l} I^{i}(q)$, with $p_{1}=1$ and $0<p_{i}<1$ (i.e., $a_{H}^{i}<c_{i}\left(p_{i}\right)<b_{H}^{i}$ ) for all $i \geq 2$.

Figure II depicts the optimal pure titles partition for $\ell=3$.

Remark (Deterministic Outputs and Lotteries) When outputs are deterministic (or can take on only a finite number of values), one can achieve the same effect

\footnotetext{
${ }^{31}$ See the proof of Theorem 12 in the Appendix, for explicit formulae for $I^{i}(p)$.
} 
by introducing lotteries on titles. Thus, in Figure II above, suppose all outputs are deterministic. Then reward the work outputs of agents $1,2,3$ with the following lotteries on titles: A with probability $p_{3}$ and $\mathrm{B}$ with probability $1-p_{3}$ for agent 3 ; $\mathrm{B}$ with probability $p_{2}$ and $\mathrm{C}$ with $1-p_{2}$ for agent 2 ; and $\mathrm{C}$ with probability $p_{3}=1$ for agent 1. For their shirk outputs award B,C,D to $3,2,1$ with probability 1 . It is evident that these lotteries will generate the same status payoffs and incentives as in Figure II.

\subsection{Wages Alone}

We assume that all agents have the same concave utility function $u$ for wages. We also assume that $u$ is continuous and strictly monotonic, with $u(x) \longrightarrow \infty$ as $x \longrightarrow \infty$. If titles confer no status, and the owner must motivate his employees only by wages, then he must ensure that the wage incentive to work for each employee of type $i$ is enough to overcome his disutility of working, i.e.

$$
\int u(w(x)) f_{i}(x) d x-\int u(w(x)) g_{i}(x) d x \geq d_{i}
$$

It is perfectly clear what needs to be done. The owner would then simply set $p_{i}=1$ for all $i=1, \ldots, \ell$ and compensate each employee for precisely his disutility when he switches from shirk to work. Since wages must be monotonic in output, this implies that an optimal pure wage schedule is a step function which pays $w_{0}$ on $\left[0, a_{H}^{1}\right)$ and $w_{i}$ on $\left[a_{H}^{i}, a_{H}^{i+1}\right)$, where $w_{0}<w_{1}<\ldots . .<w_{l}$ are defined recursively as follows, starting with $w_{0}$ :

$$
\begin{aligned}
w_{0} & =w_{\text {min }} \\
u\left(w_{i}\right)-u\left(w_{i-1}\right) & =d_{i}
\end{aligned}
$$

(Our assumptions on $u$ guarantee the existence of such a wage schedule for any $\left.w_{\min }, d_{1}, \ldots, d_{l}.\right)$ Without status, wages rise with ability, but in increments determined entirely by the utility of wages and the disutility of effort. When employees are risk neutral, we have that the wage ladder starts at $w_{0}=w_{\min }$ and jumps by $d_{i}$ at output level $a_{H}^{i}$. When there is strict risk-aversion (i.e., $u$ is strictly concave), the jumps escalate as we go up the wage ladder, compared to the risk neutral case.

Figure III depicts the optimal pure wage schedule for $\ell=3$.

\subsection{Wages and Titles Together}

Once again we ask the question: given that the owner can use both titles and wages as incentives, how should he deploy them together? As we have just seen, solving for them separately often leads to very different partitions. For example, if $d_{i}=d$ for all $i$, cuts are in the interior of $J_{H}^{i}$ for pure titles and on its boundary for pure wages. Superposing the wage schedule onto the pure titles schedule, as in the homogenous case, is in general not possible. 


\section{The Optimal Pure Wage Schedule}

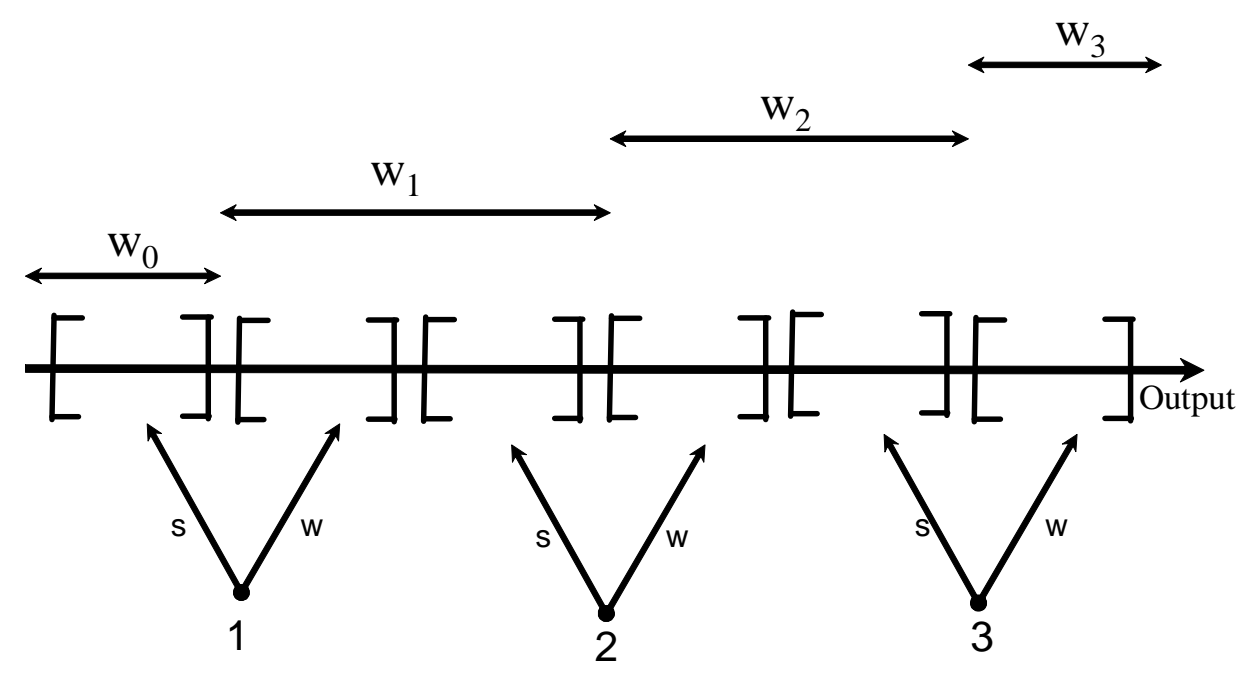

Figure III

When wielding wages and titles together, should the owner use money and status in equal proportions for all employees? Or should he, for example, reserve status mostly for higher employee types? And does our answer depend on whether the wages are secret or public?

\subsubsection{Secret Wages}

The owner's optimization problem is given below for the case of secret wages. He seeks to minimize his wage bill, subject to incentivizing everyone to work.

At first glance one might think that the highest type, who will necessarily wind up with the highest status payoff, ought to be motivated by status, while the lowest type, who will necessarily wind up with the least status payoff, will need to be motivated by money. But quite the opposite is true. As we said in the introduction, shame is the flip side of honor. So status considerations apply at both ends. Furthermore it is the change in status payoff (or money payoff) upon switching from shirk to work that counts for incentive, not the absolute payoffs.

Since wages have to be monotonic, giving a raise to the bottom end will push wages up for all, creating a huge wage bill for the owner. It is to his advantage to make the initial rung of the wage staircase as low as possible. He can achieve this by incentivizing the lowest type as much as possible via status, so that the wage incentive needed for the lowest ability employee is small. When wages are secret, the employer can indeed concentrate all the status incentive on the lowest type.

We require 
The Necessity-of-Wages Assumption (Disparate Case):

At any feasible wage-title schedule $(p, w)$, each agent must get a positive wage incentive (in addition to his status incentive) ${ }^{32}$.

Theorem 12 (Optimal Wage-Title Schedule with Secret Wages and Risk Neutrality) Suppose there are $\ell$ disparate risk-neutral types. Then the optimal wage-title schedule, when wages can be kept secret, has just two titles, the low title for outputs below $a_{H}^{1}$ (that would be given to a person of the lowest type were he to shirk) and the high title for all outputs above $a_{H}^{1}$. Thus despite the freedom to hand out titles costlessly, that would increase total status incentives, the owner should award every worker the same high title. All workers above those of type 1 are incentivized by the secret wages alone. So the wage schedule is a step function where the jump from $w_{i-1}$ to $w_{i}$ takes place at $a_{H}^{i}$ and

$$
\begin{aligned}
& w_{0}=w_{\min } \\
& w_{1}=w_{0}+d_{1}-\left(N_{1}-1+N_{2}+\ldots+N_{l}\right) \\
& w_{i}=w_{i-1}+d_{i}
\end{aligned}
$$

This solution is reminescent of a club, whose membership is restricted to those who produce output above a threshhold, but once in, all members enjoy the same title (even though they may secretly be getting different perquisites).

We saw that the optimal pure wage schedule is a ladder. In the risk neutral case, the optimal secret wage schedule with titles is exactly the same except that the whole wage ladder is shifted down by $\left(N_{1}-1+N_{2}+\ldots+N_{l}\right)$, which is equal to the enormous status incentive created for the lowest type by the two titles (the shirker of the lowest type getting the low title and all other outputs the high title). In short, the lowest type is incentivized as much as possible by status, while the others are motivated by wages alone.

Figure IV depicts the optimal Wage-Titles Schedule with secret wages for $\ell=3$.

\subsubsection{Public Wages}

The owner's optimization problem is exactly the same as for secret wages, except for the added constraint that wages must be measurable with respect to titles, i.e., must be constant across all outputs that are awarded the same title.

We shall see that, unlike the case of homogeneous employees, the optimal reward schedule does not arise by a simple superposition of the solutions for titles alone and wages alone. There is a more intricate interplay between wages and titles. Wages will now depend on the population distribution of employees $N_{1}, \ldots, N_{\ell}$, as well as

\footnotetext{
${ }^{32}$ This is guaranteed if, for example, $N_{i-1}+N_{i}+N_{i+1}<d_{i}$ for all $i$ (with $N_{0}=N_{\ell+1}=0$ ). Would anybody work for free, just for the status of coming ahead of all his peers?
} 


\section{The Optimal Wage-Title Schedule: Secret Wages}

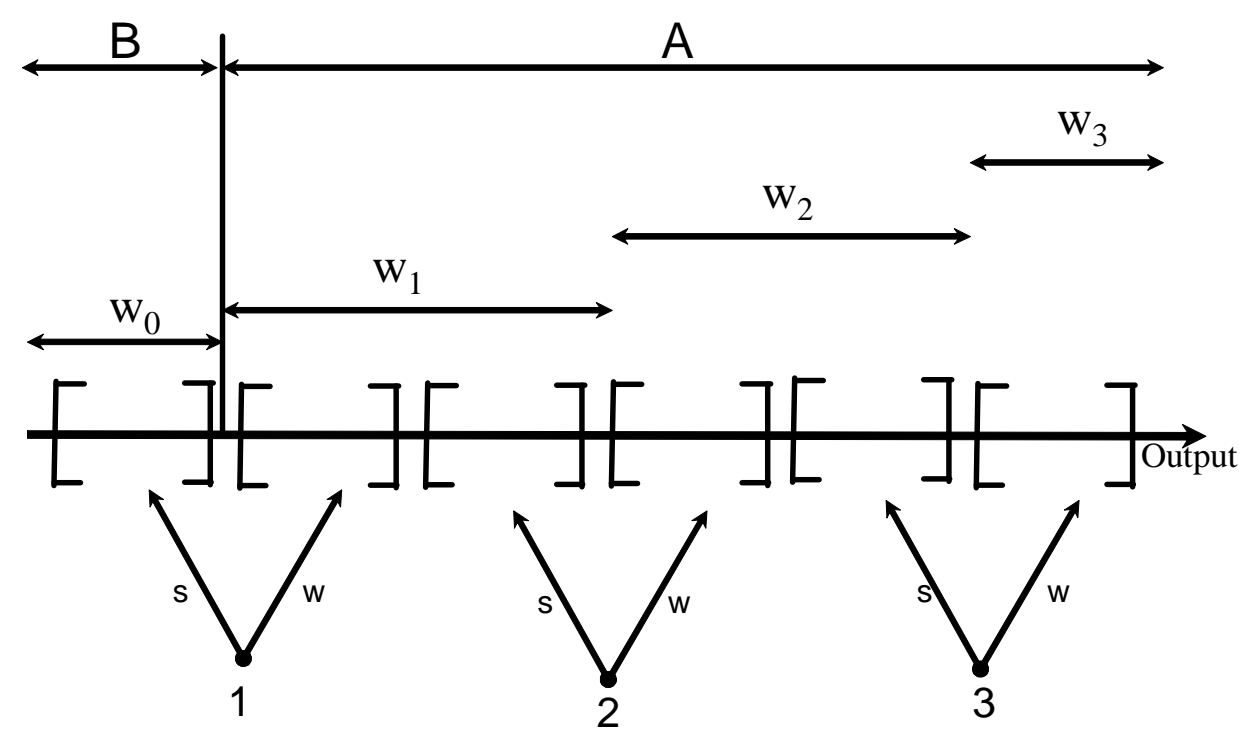

Figure IV

the disutilities of effort $d_{1}, \ldots, d_{\ell}$. But some features stand out independent of the $N_{i}$ and $d_{i}$. The most dramatic change to the wage schedule is that now a tiny elite of top performers will be given exorbitant wages. The title partition will also change. For $1 \leq i \leq \ell-1$, it is optimal to set $p_{i}=1$ so that each of those types surely gets a higher title by working. Thus the cells $C_{i}$ of the optimal title partition are as follows: $C_{0}=J_{L}^{1}$ and, for $1 \leq i \leq \ell-2, C_{i}=J_{H}^{i} \cup J_{L}^{i+1}$, and $C_{\ell-1}=J_{H}^{\ell-1} \cup J_{L}^{\ell} \cup\left[J_{H}^{\ell} \backslash J_{H}^{\ell}\left(p_{\ell}\right)\right]$, and $C_{\ell}=J_{H}^{\ell}\left(p_{\ell}\right)$. The set $C_{\ell}=J_{H}^{\ell}\left(p_{\ell}\right)$ includes just the outputs at the top end of $J_{H}^{\ell}$ whose probability is $p_{\ell}$. In the optimal public-wage \& title schedule, $p_{\ell}$ is not 1 but is close to 0 , meaning that only a tiny fraction consisting of the ultra productive employees of type $\ell$ will be awarded the topmost title when they work, while the majority of them will be pooled with the second best type $\ell-1$. The optimal publicwage \& title schedule is thus vastly different from the optimal pure titles schedule and from the optimal pure wage schedule.

Figure V depicts the optimal Wage-Titles Schedule with public wages for $\ell=3$.

The CEO is picked by lottery from the senior managing directors (type $\ell$ ). Contrary to what one might have guessed, the type $\ell$ workers are motivated almost entirely by the chance of the huge money payoff of the CEO and not by status. On the other hand, the very next tier of managing directors (type $\ell-1$ ) are motivated heavily by titles. Unlike all the other workers, by working hard they will come equal not just with all of their own type but also with virtually all of the type above them. To sum up, the top tier is motivated by money, the next is motivated as much as possible by status, and the rest by an even mix of status and money.

To ease the formal exposition of the foregoing discussion, we make the 


\section{The Optimal Wage-Title Schedule: Public Wages}

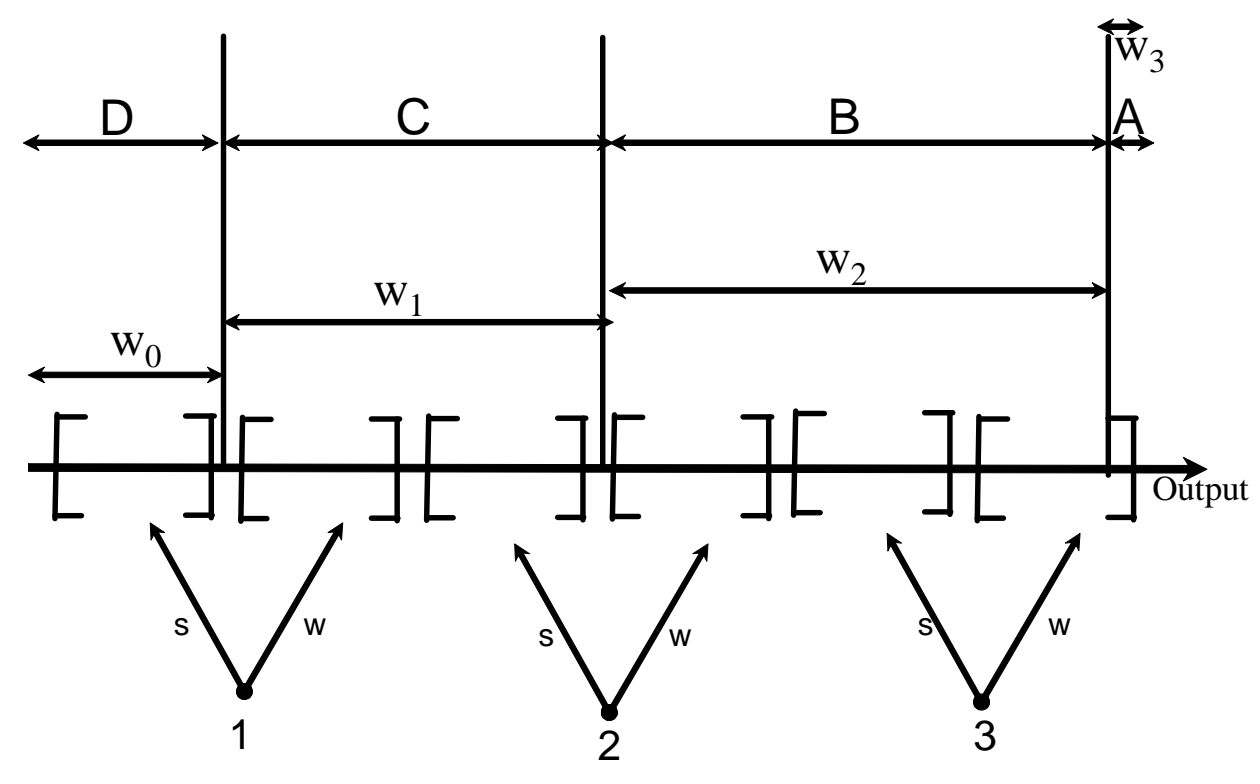

Figure $\mathrm{V}$

\section{Differentiability Assumption:}

$u$ is continuously differentiable ${ }^{33}$ (in addition to being concave and strictly monotonic).

More substantially we shall assume that agents become risk-neutral when their wealth is sufficiently large. Precisely, we have the condition below (which is automatically satisfied when $u$ is linear):

\section{Asymptotic Risk Neutrality Assumption:}

(1) $u$ becomes asymptotically risk-neutral, i.e., the derivative $u^{\prime}(w)=\lambda$ for some constant $\lambda$ whenever $w$ exceeds a threshold $w^{\tau}$.

(2) $w_{\ell-2}>w^{\tau}$ at any feasible wage-title schedule $(P, w)$,

Part (2) requires disutilities to be sufficiently more than status incentives (cumulatively across the types) so that, by the time the first $\ell-2$ agents have been given the requisite wage increases, the threshold $w^{\tau}$ is crossed. Note that if $d=\left(d_{1}, \ldots, d_{\ell}\right) \longrightarrow$ $\infty$,then $w=\left(w_{1}, \ldots, w_{\ell}\right) \longrightarrow \infty$ in order to keep $(p, w)$ feasible; so $(2)$ is automatic for large enough $d$. Tighter sufficient conditions can easily be stated in terms of the exogenous data $d, u,\left(N_{i}\right)_{i=1}^{\ell}$ of the model in order to guarantee (2), but we leave this to the reader.

Finally we assume that wages are bounded above. We did not need such an assumption previously, but the possibility of paying an arbitrarily large wage with very small probability has now become relevant.

\footnotetext{
${ }^{33}$ This is not essential and Theorem 12 below holds with just concavity of $u$. Its proof is exactly the same but with left (right) derivatives of $u$ used to estimate decreases (increases) in $u$.
} 


\section{Maximum Wage Assumption:}

Wages can never exceed $M$, where $M$ is large enough so that $u(M)-u\left(w_{\min }\right)>$ $\sum_{i=1}^{\ell} d_{i}$. In case the asymptotic risk neutrality assumption holds, assume $M$ is large enough that $\lambda\left(M-w_{\min }\right)>\sum_{i=1}^{\ell} d_{i}$.

Theorem 13 (Exorbitant Elite Wages): Let there be $\ell$ disparate types of workers, with $N_{i} \geq 1$ of each type $i=1, \ldots, \ell$. Suppose the Necessity of Wages and the Differentiability and the Maximum Wage Assumptions hold. Then at any optimal wage-title schedule $(p, w)$, we have $p_{i}=1$ for all $i=1, \ldots, \ell-1$, so that any two workers of the same type below $\ell$ get the same status and wage.

Next assume that the Asymptotic Risk Neutrality Assumption also holds. Then there is a unique optimal status partition and wage schedule $(p, w)$, with $p_{\ell} \leq\left(\lambda w_{\min }+\right.$ $\left.\sum_{i=1}^{\ell} d_{i}\right) / \lambda M<1$, and $w_{\ell}=M$. Thus for large $M, p_{\ell}$ is very small and a tiny elite $p_{\ell} N_{\ell}$ out of the highest type $\ell$ is paid the exorbitant salary $M$, while the rest of their type obtain the same status and pay as type $\ell-1$. Thus type $\ell$ employees are motivated almost entirely by wages alone.

Theorem 13 gives an explanation for the exorbitant pay often seen at the very top of some real world hierarchies. It is cheaper to incentivize the managing directors of type $\ell-1$ as much as possible via status rather than wages. To achieve this they must be able to get the same status as most of the senior managing directors of type $\ell$, if they work hard. This fixes the wage of the latter group at the managing director's level. In order to incentivize the senior managing directors, they are given to understand that the CEO will be chosen from among their rank, and even though the chance of getting selected is small, the salary is huge. (Denoting the probability of getting the top CEO title by $\epsilon$, the status incentive of type $l$ is $\epsilon\left(N_{l-1}+(1-\epsilon) N_{l}\right)$ which is negligible compared to the wage incentive $\epsilon M$, where $M$ is the huge bonus.) The conclusion is that the top ability group is motivated almost entirely by wages alone.

This stratagem of paying a huge salary to the tiny fraction of top performers in a group is counterproductive at any level below $\ell$, because monotonicity would force the employer to pay all workers of higher type at least as much.

As status grows in importance ( $\sigma$ rises above 1 ) the pay of everyone is reduced, except for the elite performers, who continue to get the same maximum M. (Every group below the top ability group was getting some status incentive and now can get more, allowing the wage reduction.) The difference in pay between the elite performers and the rest must therefore grow, and the fraction of employees getting the same elite wage must shrink. The wage schedule gets more star like.

\subsubsection{Wage Differentials for Disparate Employees}

The conclusions about exorbitant pay for the CEO and $p_{i}=1$ for all $i=1, \ldots, \ell-1$ are quite robust; they hold regardless of the distribution of abilities $N_{1}, \ldots, N_{\ell}$, or the disutilities of work $d_{1}, \ldots, d_{\ell}$. 
But the wage differentials $w_{i}-w_{i-1}$ for $i<\ell$ do depend on the $N_{j}$ 's and $d_{j}$ 's as we shall see. For simplicity let us assume $u(w)=w$ for the rest of this section. Our analysis is based on the following corollary:

Corollary to Theorem 13: Under the conditions of Theorem 13 and with risk neutrality (i.e., $u(w)=w$ ), at the optimum wage-title schedule the title incentives are $I^{1}=N_{1}-1<I^{2}=N_{1}+N_{2}-1 ; I^{i}=N_{i-1}+N_{i}-1$ for $i=3, \ldots, \ell-2$. Also, $I^{\ell-1}=N_{\ell-2}+N_{\ell-1}+\left(1-p_{\ell}\right) N_{\ell}-1 \approx N_{\ell-2}+N_{\ell-1}+N_{\ell}-1$. Finally, $I^{\ell}=p_{\ell}\left(N_{\ell-1}+N_{\ell}-1\right) \approx 0$.

Thus for $2 \leq i \leq \ell-2$,

$$
\left(w_{i}-w_{i-1}\right)-\left(w_{i-1}-w_{i-2}\right)=\left(d_{i}-d_{i-1}\right)+N_{i-2}-N_{i} .
$$

A natural case to consider is the one where the population $N_{i}$ declines in size as the ability type increases. If disutilities do not fall as fast (i.e., if $N_{i-2}-N_{i}>d_{i-1}-d_{i}$, which occurs for example, if disutilities are constant), then we conclude from the corollary that wage differentials escalate as we go up the ability ladder from $i=2$ to $i=\ell-2$.

Another natural case arises in a population that is bell-shaped around the mean ability. When $N_{i}-N_{i-2}>d_{i}-d_{i-1}$ for small $i$ and $N_{i-2}-N_{i}>d_{i-1}-d_{i}$, for large $i$, we get a wage schedule which is first concave and then convex.

The simplest case is when $N_{i}=N \forall i$ and $d_{i}=d \forall i$. Then the wage rises steadily by a fixed step of $d+1-2 N$ until $w_{\ell-2}$, then rises by only $d+1-3 N$ to $w_{\ell-1}$, then jumps astronomically to $w_{\ell}=M$.

Remark (When public wages confer status): We could have postulated, instead of titles, that status is conferred by wages themselves: $w_{i}$ confers higher status than $w_{j}$ if, and only if, $w_{i} \geq w_{j}+\delta$ for some threshold $\delta>0$. Then our last constraint in the owner's optimization problem would read: $w_{\min }=w_{0}, w_{i}+\delta \leq w_{i+1}$ for $1 \leq i \leq \ell-1, w_{\ell} \leq M$. It is worth noting that as $d=\left(d_{1}, \ldots, d_{\ell}\right) \rightarrow \infty$, $p_{i}\left(w_{i}-w_{i-1}\right) \rightarrow \infty$ since the status incentive terms $I_{\ell}^{i}(p)$ are bounded by $N_{1}+\cdots+N_{\ell}$. Thus the constraints $w_{i}+\delta \leq w_{i+1}$ are automatically satisfied for large enough $d$ (given any $\delta$ ), and our analysis remains intact.

Participation Constraints with Risk Neutrality In the case of disparate agents, observe that no matter what the underlying partition for the wage-title schedule may be, the participation constraint (PC) is met by everyone if, and only if, it is met by an agent of type 1 . This is so because his wage utility is never more than that of the others, on account of the monotonicity of the wages in terms of the output; nor is his status utility more, since titles are also monotonic and so render it impossible for him to outrank any higher type. Thus it suffices to maintain the PC for type 1. With this in mind, consider the proof of the Exorbitant Elite Wages Theorem. Start with any wage-title schedule which incentivizes everyone to work, while also 
meeting the PC for an agent of type 1 . Now read the entire proof without change.We need only check that the PC for this agent is maintained throughout. But this is straightforward. Raising $p_{1}$ boosts both his wage utility and his status utility, so the PC continues to hold for him. Next, when we raise $p_{2}$, his status utility does go down in the amount $\varepsilon N_{2}$. But the subsequent increase of $w_{1}$ to $\tilde{w}_{1}$ raises his wage utility in precisely the same amount, so that the PC is still not violated for him. The rest of the proof proceeds without at all impacting agents of type 1 . Hence the Exorbitant Elite Wages Theorem, and its proof, hold exactly as before, with just one amendment: in the optimal wage-title schedule that we wind up with, it may be that $w_{1}$ is escalated to ensure $u\left(w_{1}\right)-N_{2}-\ldots .-N_{l}=u_{*}$ (thereby meeting the PC for agents of type 1). There is no other change. The titles-partition and the wage differentials, starting from $w_{1}$, stay exactly the same.

\section{Relative Wages and Titles}

One might wonder whether it would be easier to motivate employees by paying them relative wages, i.e., wages and titles based on how their performance ranks relative to their rivals. We can formalize this by a sequence $K=\left(K_{Z}, \ldots, K_{B}, K_{A}\right)$, where the top $K_{A}$ performers get the highest wage and title, the next $K_{B}$ get the next highest wage and title, and so on. Ties are broken randomly with equal probability.

The answer is no.

\subsection{Homogeneous Employees}

Consider any money payment schedule in which the money payment to an employee is a function of his output and the output of all the others. Conditional on his own output, the worker thereby obtains a certain expected utility of the forthcoming money payment, which is equivalent to getting some wage for certain. (Since he is risk averse, this certainty equivalent wage is actually smaller than the expected money payment the owner is making, conditional on the worker's output.) If this certainty equivalent wage (thought of as a function of the worker's output) gives him the incentive to work, then it must cost the employer at least as much as the optimal pure wage schedule derived in section 3 . Thus in our model, absolute wages cannot be beaten by relative wages or any other wage schedule, when status considerations are absent.

On the other hand, with pure status, the optimal absolute partition of the Proposition beats any relative schedule $K=\left(K_{Z}, \ldots, K_{B}, K_{A}\right)$, by Dubey-Geanakoplos $(2005,2010)$. But as we saw in the Optimal Wage-Titles Theorem, this same partition also serves for the optimal wage schedule. Thus the same absolute partition gives more status incentive than any relative schedule, and also gives more wage incentive than would be generated by any relative schedule. Since status incentives and wage incentives are additive, this absolute wage-title schedule is better than any relative schedule. 


\subsection{Disparate Employees}

Consider a general population $N=\left(N_{1}, \ldots, N_{\ell}\right)$ of $\ell$ disparate types, and any relative wage-title schedule given by $K=\left(K_{A}, K_{B}, \ldots, K_{Z}\right), K_{A}+\cdots+K_{Z}=N_{1}+\cdots+$ $N_{\ell}$. (Recall that we don't need to worry about ties since outcomes are continuously distributed). We can find an absolute wage-title schedule that creates at least the same incentives (from status and money combined), while handing out the same amount of money.

Define absolute grade intervals by the intervals $\left[x_{A}, \infty\right),\left[x_{B}, x_{A}\right)$ and so on, where the cuts $x_{\alpha}$ are defined by the maximum values solving the equations

$$
K_{A}+\cdots+K_{\alpha}=\text { Expected number of people with scores in }\left[x_{\alpha}, \infty\right)
$$

assuming everybody works. Award the relative wages and titles on these absolute intervals. It is easy to check that the absolute wage-title schedule we have defined, costs the same and creates (using the concavity of $u$ ) at least the same incentives.

\section{Incomplete Information}

We have assumed so far that every player knows the precise characteristics of every other player, in addition to his own. Our analysis can be modified very easily to accomodate incomplete information, i.e., when each player knows his own characteristics precisely, but has only a probability distribution on those of others.

First consider our model of $N$ homogeneous employees, i.e., each produces random output with the same probability density $f, g$ if he works, shirks (independently of the effort chosen by the others). In order to introduce incomplete information, let us suppose that the disutility of effort can take on many possible values $d_{1}<\ldots<d_{k}$. Nature moves first, independently picking a disutility level for everyone and revealing to each only his own. An optimal reward schedule must motivate every employee to work no matter what his disutility level may be. This is clearly equivalent to motivating an employee to switch from shirk to work when his disutility is the highest possible (i.e., is $d_{k}$ ) and when the remaining $N-1$ employees are working. Thus the optimal reward schedule we have constructed in the complete information case, when all employees have the common disutility $d_{k}$, is also the optimal schedule with incomplete information.

Next consider the case of $l$ disparate ability-types, with disutility $d_{i}$ and disjoint performance intervals $J_{L}^{i}<J_{H}^{i}$ for type $i$, as before. Here the natural game of incomplete information (that we have in mind) is as follows. Nature moves first, assigning type $i=1, \ldots, l$ randomly to everyone with probabilities that are i.i.d ${ }^{34}$ across the employees, say type $i$ is picked with probability $\theta_{i}$. Each employee comes to know his own type and not those of the others, before choosing his effort level.

\footnotetext{
${ }^{34}$ What is important is that the probabilities be independent across the employees. We suppose that they are identically distributed only for ease of notation.
} 
But the game of course is common knowledge, so each is cognizant of the probability distribution on types.

The status incentive for any employee of type $i$ is linear in the expected number of rivals of each type. When there is complete information, these numbers are deterministically given by the vector $\left(N_{1}, \ldots, N_{i-1}, N_{i}-1, N_{i+1}, \ldots, N_{\ell}\right)$. When there is incomplete information, this vector does not depend on $i$, and is always given by the expected numbers $\left(\theta_{1}(N-1), \ldots, \theta_{\ell}(N-1)\right)$. Based on this observation, the entire analysis of the disparate case can be transported from complete information to incomplete information as follows. Lemma 4 (Cuts), Lemma 5 (Optimal Pure Titles), and Lemma 6 (Cuts with Wages) all hold mutatis mutandis. There is a variant of Theorem 12 (Optimal Wage-Title Schedule with Secret Wages and Risk Neutrality) which goes as follows

Theorem 12' (Variant of Theorem 12 for Incomplete Information) Consider the setting of Theorem 12 but with incomplete information. There is an optimal secret wage-title schedule (though no longer necessarily unique) that still consists of just one cut at $a_{H}^{1}$. The formula for wages is also just the same, except that

$$
w_{1}=w_{0}+d_{1}-(N-1)
$$

Finally consider the case of public wages. A variant of Theorem 13 (Exorbitant Wages) also remains intact:

Theorem 13' (Variant of Theorem 13 for Incomplete Information) Consider the setting of Theorem 13 but with incomplete information. All optimal partitions must have $p_{i}=1$ for $1 \leq i \leq l-1$ and - with the Asymptotic Risk Neutrality Assumption - there exists an optimal schedule with $p_{l} \leq\left(\lambda w_{\min }+\sum_{i=1}^{l} d_{i}\right) / M<1$ and $w_{l}=M$.

As with secret wages, we can no longer assert uniqueness of the optimal wagetitle schedule. Thus exorbitant wages must occur with complete information and constitute one of the feasible optima if there is incomplete information. This leads us to conjecture that exorbitant wages become necessary for any information regime that is in between the two. The modeling of such information regimes and the precise formulation of the result is left to future research.

\section{References}

Auriol E. and R. Renault (2008) "Status and Incentives", RAND Journal of Economics, vol 39(1), pp. 305-326.

Brown, G., and J. Gardner, A. Oswald, and J. Qian (2008) "Does Wage Rank Affect Employees Well Being", Industrial Relations: A Journal of Economy and Society, Volume 47, Issue 3, pages 355-389. 
Becker, Gary S., Kevin M. Murphy, and Ivan Werning (2005). "The Equilibrium Distribution of Income and the Market for Status," Journal of Political Economy, 100(6): 1092-1125.

Cole, Harold L., George J. Mailath, and Andrew Postlewaite (1992). "Social Norms, Savings Behavior, and Growth," Journal of Political Economy, 113(2): 282-310. (1995). "Incorporating Concern for Relative Wealth into Economic Models," Federal Reserve Bank of Minneapolis Quarterly Review, 19(3): 12-21.

(1998). "Class Systems and the Enforcement of Social Norms," Journal of Public Economics, 70: 5-35.

Coleman, James S. (1961). The Adolescent Society, Glencoe, Illinois, Free Press.

Coleman, James S. (1990). Foundations of Social Theory, Cambridge, MA: Harvard University Press.

Corneo, Giacomo and Oliver Jeanne (1998). "Social Organization, Status and Savings Behavior," Journal of Public Economics 70: 37-51

Direr, A. (2001). "Interdependent Preferences and Aggregate Saving," Annales d'Economie et de Statistique, 297-308.

Dubey, P. and J. Geanakoplos (2005). "Incentives in Games of Status: Marking Exams and Setting Wages," Cowles Foundation Discussion Paper No. 1544, Yale University.

Dubey, P. and J. Geanakoplos (2010). "Grading Exams: 100, 99, ..., 1 or A, B, C?" Games and Economic Behavior, 69, pp 72-94.

Dubey, P., J. Geanakoplos and O. Haimanko (2013). "Prizes versus Wages with Envy and Pride," The Japanese Economic Review, 64(1): 98- 121.

Duesenberry, James S. (1949). Income, Saving and the Theory of Consumer Behavior. Cambridge: Harvard University Press.

Fehr, E. and K.M. Schmidt (1999). "A Theory of Fairness, Competition and Cooperation," Quarterly Journal of Economics, 114: 817-868.

Frank, Robert H. (1985). Choosing the Right Pond: Human Behavior and the Quest for Status. New York: Oxford University Press.

Friedman, Milton. (1953). " Choice, Chance and the Personal Distribution of Income," Journal of Political Economy, 61(4): 277-290.

Friedman, Milton.and Leonard J. Savage (1948). "The Utility Analysis of Choices Involving Risk," Journal of Political Economy, 61(4): 279-304.

Green, J.R. and N.L. Stokey (1983). "A Comparison of Tournaments and Contracts," Journal of Political Economy, 91(3): 349-364. 
Goode, William J. (1979). The Celebration of Heroes: Prestige as a Social Control System. Berkeley, University of California Press.

Hopkins, Ed and Tatiana Kornienko (2004). "Running to Keep the Same Place: Consumer Choice as a Game of Status," American Economic Review 94 (4): 1085-1107.

Lazear, E. and S. Rosen (1981). "Rank-Order Tournaments as Optimal Labor Contracts," Journal of Political Economy, 89: 841-864.

Moldovanu, B. and A. Sela (2001). "The Optimal Allocation of Prizes in Contests," American Economic Review, 91(3): 542-558.

Moldovanu, B., A. Sela and X. Shi (2007). "Contests for Status" Journal of Political Economy, 115, pp 338-363.

Park, E. (1995) "Incentive Contracting Under Limited Liability", Journal of Economics and Management Strategy, 4, 3, 477-490.

Pollak, Robert (1976). "Interdependent Preferences," American Economic Review, 66(3): 309-320.

Robson, Arthur (1992). "Status, the Distribution of Wealth, Private and Social Attitudes to Risk," Econometrica, 60(4): 837-857.

Shaked, M. and J.G. Shanthikumar (1994). Stochastic Orders and their Applications. San Diego: Academic Press.

Veblen, Thorstein (1899). The Theory of the Leisure Class. New York: Macmillan.

Weber, M. (1922). Economy and Society: an Outline of Interpretive Sociology, Berkeley, University of California Press.

\section{Appendix}

We here present proofs that did not appear in the main text.

Recall that for any subset $A \subset Q$, we let $f(A)=\sum_{x \in A} f(x)$ and $g(A)=$ $\sum_{x \in A} g(x)$.

Incentive Lemma: Let a cell $C=L \cup R$ in a partition $P$ be the union of two consecutive intervals $L<R$. If

$$
\frac{f(L)}{g(L)}<\frac{f(R)}{g(R)}, \frac{f(L)}{g(L)}=\frac{f(R)}{g(R)}, \frac{f(L)}{g(L)}>\frac{f(R)}{g(R)}
$$

then the incentive to work is strictly improved, left unchanged, strictly worsened (respectively) by splitting $C$ into $L$ and $R$.

Proof: Splitting $C$ changes the incentive to work by $f(R) g(L)-f(L) g(R)$. 
Merger Lemma: Suppose inside domination holds separately on two consecutive intervals $L<R$. If

$$
\frac{f(L)}{g(L)} \geq \frac{f(R)}{g(R)}
$$

then inside domination holds on the single cell $L \cup R$.

Proof: Take an arbitrary cut of $R$ into $R_{-}<R_{+}$. By inside dominaton $Y \succsim X$ on $R$,

$$
\frac{f\left(R_{-}\right)}{g\left(R_{-}\right)} \geq \frac{f\left(R_{+}\right)}{g\left(R_{+}\right)}
$$

Putting this together with the hypothesized inequality immediately gives

$$
\frac{f\left(L \cup R_{-}\right)}{g\left(L \cup R_{-}\right)} \geq \frac{f\left(R_{+}\right)}{g\left(R_{+}\right)}
$$

If the cut occurs inside $L$, an analogous proof works. If the cut divides $L$ from $R$, there is nothing to prove.

Splitting Lemma: Suppose inside dominaton holds on a cell $C=L \cup R$ that is the union of two consecutive intervals $L<R$. If

$$
\frac{f(L)}{g(L)} \leq \frac{f(R)}{g(R)}
$$

then inside dominaton holds separately on each of the cells $L, R$.

Proof: Take an arbitrary cut of $R$ into $R_{-}<R_{+}$. By inside dominaton $Y \succsim X$ on $L \cup R$,

$$
\frac{f\left(L \cup R_{-}\right)}{g\left(L \cup R_{-}\right)} \geq \frac{f\left(R_{+}\right)}{g\left(R_{+}\right)}
$$

It follows from this and the inequality hypothesized that

$$
\frac{f\left(R_{-}\right)}{g\left(R_{-}\right)} \geq \frac{f\left(R_{+}\right)}{g\left(R_{+}\right)}
$$

proving that $R$ satisfies inside dominaton. A similar argument applies to $L$.

Though uniform domination is stronger than domination, the two become equivalent when they are opposed:

Constant Ratio Lemma: If $X$ uniformly dominates $Y$ on the interval $[c, d]$ and $Y$ dominates $X$ on $[c, d]$, then

$$
\frac{f(a)}{g(a)}=\frac{f(b)}{g(b)}
$$

for all $a, b \in[c, d]$. 
Proof: $\quad$ Since $X \succsim_{U} Y$ on $[c, d]$,

$$
\frac{f(b)}{g(b)}
$$

is weakly increasing in $b \in[c, d]$. However, since $Y \succsim X$ on $[c, d]$, taking a cut just before the last element $d$ gives

$$
\frac{\operatorname{Pr}(X \in[c, d))}{\operatorname{Pr}(Y \in[c, d))} \geq \frac{f(d)}{g(d)}
$$

These two conditions are compatible only if $f(b) / g(b)$ is constant for $b \in[c, d]$.

Constant Incentive Lemma: Suppose the partition $\mathcal{P}$ satisfies inside dominaton and that the partiton $\mathcal{P}^{\prime}$ satisfies outside domination. If $\mathcal{P}^{\prime}$ is a refinement of $\mathcal{P}$, then $\frac{f(A)}{g(A)}$ is a constant across the cells $A$ of $P^{\prime}$ that subdivide any cell $C$ of $\mathcal{P}$; consequently both partitions give the same status incentive.

Proof: On any cell $C$ of $\mathcal{P}, Y$ dominates $X$ by the inside domination hypothesis on $\mathcal{P}$. But across the cells of $\mathcal{P}^{\prime}$ that subdivide this cell $C$ of $\mathcal{P}, X$ uniformly dominates $Y$ by the outside domination hypothesis on $\mathcal{P}^{\prime}$. Hence, by the Constant Ratio Lemma, $\frac{f(A)}{g(A)}$ is a constant across the cells $A$ of $\mathcal{P}^{\prime}$ that subdivide $C$. Therefore by the Incentive Lemma, incentives are the same for $\mathcal{P}$ and $\mathcal{P}^{\prime}$

Tail Lemma: Let $P$ be a partition of $Q$ into consecutive cells $\{\ldots, E<B, \ldots\}$ that satisfies inside and outside domination with respect to $X$ and $Y$. Suppose that $D \subset E$ is a right tail segment of $E$ and $C \subset B$ is a left tail segment of $B$. Then

$$
\frac{f(D)}{g(D)} \leq \frac{f(C)}{g(C)}
$$

Proof: From outside domination we know that

$$
\frac{f(E)}{g(E)} \leq \frac{f(B)}{g(B)}
$$

From inside domination we know that

$$
\frac{f(D)}{g(D)} \leq \frac{f(E)}{g(E)} \text { and } \frac{f(B)}{g(B)} \leq \frac{f(C)}{g(C)}
$$

proving the lemma

Join-Meet Lemma: Suppose the partitions $P$ and $P^{\prime}$ each satisfy inside and outside domination. Then so do their join and meet. 
Proof: Let $B^{\vee}<A^{\vee}$ be any two consecutive cells of the join $\mathcal{P} \vee \mathcal{P}^{\prime}$. The cut between them must come from one of the partitions, hence by the Tail Lemma

$$
\frac{f\left(B^{\vee}\right)}{g\left(B^{\vee}\right)} \leq \frac{f\left(A^{\vee}\right)}{g\left(A^{\vee}\right)}
$$

Thus $f / g$ is increasing over the cells of $\mathcal{P} \vee \mathcal{P}^{\prime}$ as we move to the right, proving outside domination for $\mathcal{P} \vee \mathcal{P}^{\prime}$. By the Splitting Lemma, inside domination holds for each cell of $\mathcal{P} \vee \mathcal{P}^{\prime}$ contained in any cell of $\mathcal{P}$ or $\mathcal{P}^{\prime}$, and hence it holds in every cell of $\mathcal{P} \vee \mathcal{P}^{\prime}$. Thus inside and outside domination hold for the join.

By the Constant Ratio Lemma, inside domination on $\mathcal{P}$ and inside domination on $\mathcal{P}^{\prime}$ then imply that $f / g$ is constant over all the cells from $\mathcal{P} \vee \mathcal{P}^{\prime}$ that lie in the same cell of $\mathcal{P}$ and over all the cells from $\mathcal{P} \vee \mathcal{P}^{\prime}$ that lie in the same cell of $\mathcal{P}^{\prime}$. Hence $f / g$ is constant over all the cells from $\mathcal{P} \vee \mathcal{P}^{\prime}$ that lie in any cell of $\mathcal{P} \wedge \mathcal{P}^{\prime}$. Hence by the merger lemma, the inside condition holds on each cell of $\mathcal{P} \wedge \mathcal{P}^{\prime}$. The outside condition for the meet follows from the simple fact that $f / g$ is rising across the cells of the join.

\section{Proof of Theorem 1 (Inside-Outside Condition):}

Proof of Necessity: If the inside condition is violated when some cell $C$ of an optimal partition $\mathcal{P}$ is cut into two consecutive cells $L<R$, then (by the incentive lemma), splitting $C$ improves incentives, contradicting the optimality of $\mathcal{P}$. Similarly, if $f / g$ strictly falls across two consecutive cells of $\mathcal{P}$, then (by the incentive lemma) merging them strictly increases incentives. Hence $f / g$ must be (weakly) increasing across all cells of $\mathcal{P}$.

Proof of Sufficiency: Suppose $\mathcal{P}$ satisfies the inside and outside conditions. Since $Q$ is finite, there must trivially exist an optimal partition $\bar{P}$. By the necessity proof, $\bar{P}$ satisfies the inside and outside conditions. By the Join-Meet Lemma and the Constant Incentive Lemma, the meet $\mathcal{P} \wedge \overline{\mathcal{P}}$ and the join $\mathcal{P} \vee \overline{\mathcal{P}}$ give the same incentive as both $\mathcal{P}$ and $\bar{P}$, proving that $\mathcal{P}$ is also optimal.

Proof of Theorem 2 (Lattice Structure): By Theorem 1 and the Join-Meet lemma, the optimal title partitions form a lattice as claimed. Let $C$ be a cell in the maximal optimal partition $\mathcal{P}^{*}$. If some cut of $C$ into consecutive intervals $L<R$ leaves the ratios $f(L) / g(L)=f(R) / g(R)$, then (by the incentive lemma) it also leaves incentives unchanged when $C$ is split into $L$ and $R$, contradicting the maximality of $\mathcal{P}^{*}$. Hence the inside condition must always hold strictly on cells of $\mathcal{P}^{*}$.

Next, let $L<R$ be consecutive cells of the minimal optimal partition $\mathcal{P}_{*}$. Again, if $f(L) / g(L)=f(R) / g(R)$, then (by the incentive lemma) it also leaves incentives unchanged when $L$ and $R$ are merged into $C=L \cup R$, contradicting the miminality of $\mathcal{P}_{*}$. Hence the outside condition must always hold strictly on cells of $\mathcal{P}_{*}$.

Consider a partition $\mathcal{P}$ obtained via any subset of the cuts of the join $\mathcal{P}^{*}$ that includes all the cuts of the meet $\mathcal{P}_{*}$. By the constant ratio lemma, $f / g$ is constant 
across the cells of $\mathcal{P}^{*}$ that lie in the same cell of $\mathcal{P}_{*}$ Hence by the merger lemma, the new partition $\mathcal{P}^{*}$ must also satisfy the inside condition on each of its cells. It obviously inherits the outside condition from $\mathcal{P}^{*}$. Hence $\mathcal{P}$ is optimal by Theorem

1.

Proof of Lemma 1 (Coarse Partition): If there were a cut between $x$ and $y$, then by the Tail Lemma we would have

$$
\frac{f(x)}{g(x)} \leq \frac{f(y)}{g(y)}
$$

a contradiction.

Proof of Lemma 2 (Fine Partition): This is an immediate corollary of Theorem 1.

Proof of Lemma 3 (Unicity of the Lattice): For almost all $f$ and $g$, it is clear that

$$
\frac{f(A)}{g(A)} \neq \frac{f(B)}{g(B)}
$$

for any two unequal intervals $A, B$ of $Q$. By the constant ratio lemma, any optimal partition that is finer than the minimal optimal partition $\mathcal{P}_{*}$ must produce an equality on cells that lie in the same cell of $\mathcal{P}_{*}$.

For what follows it will be useful to recall a standard property of stochastic dominance (see e.g. Shaked-Shanthikumar 1994).

Lemma (Dominance Increases Expectation) : Suppose that $Y$ dominates $X$ on the interval $C$, and that $f(C) g(C)>0$. If $\phi: C \rightarrow \mathbb{R}$ is any monotonic function, then

$$
\frac{1}{g(C)} \sum_{x \in C} \phi(x) g(x) \geq \frac{1}{f(C)} \sum_{x \in C} \phi(x) f(x)
$$

where the inequality is strict if $\phi$ is not constant, and $Y$ strictly dominates $X$.

Proof: The proof is by induction on the number of values $\phi$ takes on. If it takes on just one value, there is nothing to prove. So assume the theorem is true if $\phi$ takes on $k$ values. Now consider a $\phi$ which takes on $k+1$ values $c_{1}<\ldots<c_{k}<c_{k+1}$. Let $C^{\prime}$ be the right tail of $C$ on which $\phi$ takes its maximal value $c_{k+1}$. Define $\phi^{\prime}$ by leaving it unchanged on $C \backslash C$ l and reducing $\phi$ on $C$ ' from $c_{k+1}$ to $c_{k}$.. From the domination hypothesis

$$
\frac{1}{g(C)}\left(c_{k+1}-c_{k}\right) g\left(C^{\prime}\right) \leq \frac{1}{f(C)}\left(c_{k+1}-c_{k}\right) f\left(C^{\prime}\right)
$$


where the inequality is strict if $g$ strictly dominates $f$. By the inductive assumption

$$
\begin{aligned}
\frac{1}{g(C)} \sum_{x \in C} \phi(x) g(x) & =\frac{1}{g(C)} \sum_{x \in C} \phi^{\prime}(x) g(x)+\frac{1}{g(C)}\left(c_{k+1}-c_{k}\right) g\left(C^{\prime}\right) \\
& \leq \frac{1}{f(C)} \sum_{x \in C} \phi^{\prime}(x) f(x)+\frac{1}{f(C)}\left(c_{k+1}-c_{k}\right) f\left(C^{\prime}\right)=\frac{1}{f(C)} \sum_{x \in C} \phi(x) g(x)
\end{aligned}
$$

where again the inequality is strict if $g$ strictly dominates $f$.

Proof of Theorem 3 (Compatibility of Pure Wages and Pure Titles) Let $w: Q \rightarrow\left[w_{\min }, \infty\right)$, be any wage schedule in $\mathcal{W}$ that is not measurable wrt the maximal optimal titles partition $\mathcal{P}^{*}$. We shall construct another wage schedule $w^{*} \in$ $\mathcal{W}$ that is measurable wrt the maximal optimal titles partition $\mathcal{P}^{*}$ and creates a strictly higher incentive to work than $w$ does.

For each cell $C$ of $\mathcal{P}^{*}$, define the constant wage $w_{C}$ such that

$$
u\left(w_{C}\right)=\frac{1}{f(C)} \sum_{q \in C} f(q) u(w(q))
$$

(If $f(C)=0$, set $w_{C}=\max \{w(x): x<C\}$; otherwise, $w_{C}$ exists because of the intermediate value theorem and the continuity of $u$.) Since $u$ is concave and monotonic,

$$
w_{C} \leq \frac{1}{f(C)} \sum_{q \in C} f(q) w(q)
$$

for every $C \in \mathcal{P}^{*}$. Hence the function $w^{*}$, made by patching $w_{C}$ across all $C \in \mathcal{P}^{*}$, is no more costly for the employer than $w$. By construction, the worker gets the same utility payoff from both wage schedules. It remains to show that the shirker gets a strictly lower utility payoff in $w^{*}$ than in $w$, implying that $w^{*}$ creates strictly more incentive to work than $w$.

From Theorem 2 (Lattice Structure), we know that $g$ strictly dominates $f$ on $C$. Since $u$ and $w$ are both monotonic, so is $u(w(q))$, and hence by the Lemma above (Dominance Increases Expectation) when $f(C) g(C)>0$

$$
u\left(w_{C}\right)=\frac{1}{f(C)} \sum_{q \in C} f(q) u(w(q)) \leq \frac{1}{g(C)} \sum_{q \in C} g(q) u(w(q))
$$

with strict inequality on cells $C$ on which $w$ is not constant. So the change in the shirker's utility payoff on any $C$ by moving from $w$ to $w^{*}$ is

$$
\begin{gathered}
\sum_{q \in C} g(q)\left(u\left(w_{C}\right)-u(w(q))=\right. \\
g(C) u\left(w_{C}\right)-\sum_{q \in C} g(q) u(w(q)) \leq 0
\end{gathered}
$$

from the above when $f(C) g(C)>0$, and trivially when $g(C)=0$, and also when $f(C)=0$, because in this last case $w_{C} \leq w(q)$ for all $q \in C$. The change is strict on 
every cell $C$ on which $w$ is not constant. Thus if $w$ is not measurable with respect to $\mathcal{P}^{*}$, it is not optimal.

To prove the second half of the theorem, note first that feasible wage schedules form a compact subset of the finite dimensional space $\mathbb{R}^{Q}$. By continuity of the total wage bill, an optimal solution $w$ exists, which by our proof is measurable with respect to $\mathcal{P}^{*}$. By averaging as above over cells of $\mathcal{P}_{*}$ instead of $\mathcal{P}^{*}$, we obtain a wage schedule $w_{*}$ on $\mathcal{P}_{*}$. By construction the expected utility to the worker remains the same, and the wage bill does not go up. But since $f / g$ is constant over the cells of $\mathcal{P}^{*}$ which constitute any given cell of $\mathcal{P}_{*}$, the expected utility to the shirker has also remained constant. Thus the incentive to work is unchanged. This proves that $w_{*}$ is also an optimal wage schedule.

Proof of Theorem 4 (Minimum Wage with Risk Aversion): By the outside condition, $f\left(C_{i}\right) / g\left(C_{i}\right)$ is weakly increasing in $i$. Hence $f\left(C_{i}\right) \leq g\left(C_{i}\right)$ for all $i \leq k$. As just shown, the owner is paying a constant wage $w_{i}$ on each cell $C_{i}$. Reducing all $w_{i}$ to $w_{\text {min }}$ for all $i \leq k$ strictly reduces the wage bill, unless already $w_{i}=w_{\min }$ for all $i \leq k$, and does not decrease the incentive to work

Proof of Corollary to Theorem 4 (Uniqueness with Strict Risk Aversion) From Theorem 3 (Compatibility of Wages and Titles) every optimal schedule $w$ musr be constant on the cells $C_{1}, \ldots, C_{L}$ of $\mathcal{P}^{*}$. Hence we may write the owner's optimization problem as

$$
\begin{aligned}
\min _{w \in \mathbb{R}^{L}} & \sum_{\ell=1}^{L} f\left(C_{\ell}\right) w_{\ell} \\
\text { s.t. } & \sum_{\ell=1}^{L}\left[f\left(C_{\ell}\right)-g\left(C_{\ell}\right)\right] u\left(w_{\ell}\right) \geq d \\
w_{L+1} & \equiv \infty>w_{L} \geq \ldots \geq w_{1} \geq w_{\min } \equiv w_{0}
\end{aligned}
$$

By Theorem 4 there is a $k$ such that $w_{i}=w_{\min }$ for all $i \leq k$; and $f\left(C_{\ell}\right)>g\left(C_{\ell}\right)$ for all $\ell>k$. If there are two distinct solutions, then at least one of them has $w_{L}>w_{\min }$, otherwise both would be identically $w_{\min }$. Now the half-half convex combination $w^{*}$ (of the two solutions) trivially satisfies the bottom sequence of linear inequalities and leaves the minimand unchanged. Furthermore, since the two solutions agree (and are equal to $\left.w_{\min }\right)$ at every $\ell$ with $f\left(C_{\ell}\right)-g\left(C_{\ell}\right) \leq 0$, the combination $w^{*}$ must satisfy the first (incentive) constraint strictly on account of the strict concavity of $u$. We can lower slightly all the wages in $w^{*}$ that are strictly above $w_{\min }$, maintaining all the constraints but lowering the wage bill, a contradiction.

Proof of Theorem 8 (Optimal Wage-Title Schedule): Consider the pair $\left(\mathcal{P}^{*}, w^{*}\right)$ where $\mathcal{P}^{*}$ is the maximal optimal titles partition and $w^{*}$ is any solution to the pure wage problem with $d^{*}=d-I_{\sigma}\left(\mathcal{P}^{*}\right)$ in place of $d$. Then by Theorem 5 (Wage Structure with Risk Aversion), $w^{*}$ is measurable with respect to $\mathcal{P}^{*}$. Then 
$\left(\mathcal{P}^{*}, w^{*}\right)$ is feasible, hence the total wage bill in $w^{*}$ is at least as high as the total wage bill in the optimal $w$. It follows that $I_{\sigma}(w) \leq I_{\sigma}\left(w^{*}\right)$, since $w^{*}$ is an optimal pure wage schedule. $\mathcal{P}$ generates status incentive $I_{\sigma}(\mathcal{P}) \leq I_{\sigma}\left(\mathcal{P}^{*}\right)$, since $\mathcal{P}^{*}$ is an optimal titles partition. Since $(\mathcal{P}, w)$ is optimal, we must have that the joint incentive $I_{\sigma}(\mathcal{P})+I(w) \geq d=I_{\sigma}\left(\mathcal{P}^{*}\right)+I\left(w^{*}\right)$, and hence that $I_{\sigma}(\mathcal{P})=I_{\sigma}\left(\mathcal{P}^{*}\right)$ and $I(w)=I\left(w^{*}\right)$. Thus $\mathcal{P}$ solves the pure titles problem and $w$ solves the pure wage problem for $d^{*}=d-I_{\sigma}\left(\mathcal{P}^{*}\right)=d-I_{\sigma}(\mathcal{P})$.

For generic $\mathrm{f}$ and $\mathrm{g}$, Lemma 3 (Unicity of the Lattice) implies that $\mathcal{P}=\mathcal{P}^{*}=\mathcal{P}_{*}$

Proof of Theorem 9 (Star Wages): Immediate from Theorem 8 (Optimal WageTitle Schedule) and Theorem 6 (Trigger Wage with Risk Neutrality)

Proof of Theorem 10 (Status Creates Star-Like Wages): Raising $\sigma$ is tantamount to decreasing $d$ in the pure wage problem, because the required wage incentive is given by $d-\sigma I^{S}$. The result now follows from Theorem 7 (Trigger-Like Wages with Risk Aversion)

Now we turn to the disparate case. We begin by proving a lemma:

Lemma 5 (Cuts): Suppose there are $\ell$ disparate types, and a given title partition. Then there is another title partition, with (1) the lowest cut at $a_{H}^{1}$, (2) at most one cut in every $J_{H}^{i}, i \geq 1$, and (3) no other cuts, which improves (or leaves unchanged) the status incentive to work of every employee.

Proof of Lemma 5 (Cuts): Let us consider a title partition defined by a finite set of cuts. Since in equilibrium there is nobody in any of the intervals $\left(b_{H}^{i}, a_{H}^{i+1}\right)$, any cut in such an interval can be moved to $a_{H}^{i+1}$ without hurting the status payoff of any worker, and leaving unchanged or perhaps hurting the status payoff of the unilateral shirker of type $i$. If there is not a cut at $a_{H}^{1}$, add it. This reduces (or leaves unchanged) the status payoff of the shirker of type 1 without changing the status payoff (under work or shirk) of anybody else, because in equilibrium there is nobody below $a_{H}^{1}$.

Now suppose there is a cut at $a_{H}^{1}$ and at least two cuts in some $J_{H}^{i}$. Remove the highest of all the cuts in $J_{H}^{i}$. Notice first that this does not affect the status payoff of any (worker or shirker) of type $j<i$, or of the shirker of type $i$, since they all come below the second highest cut in $J_{H}^{i}$ anyway. Thus the status incentives of employees of types $j<i$ are unaffected. The status payoff of any shirker of type $j>i$ must go down by at least as much as that of the worker of the same type; hence their status incentives cannot decrease. Finally, the status payoff of a worker of type $i$ can only go up. Against workers of his own type, he always gets expected status payoff of zero (by symmetry), and eliminating the cut increases (or leaves unchanged) his probability of coming equal with workers of higher type. This proves the lemma by iteratively removing all but the lowest cut from each interval $J_{H}^{i}$.

Recall that the cut in $J_{H}^{i}$ is denoted by $c_{i}$, and that $p_{i}=\int_{c_{i}}^{b_{i}^{H}} f_{i}(x) d x$ is the probability of the upper tail $J_{H}^{i}\left(c_{i}\right)=\left\{q \in J_{H}^{i}: q \geq c_{i}\right\}$. In view of the cuts 
lemma, we concentrate our attention on partitions $\mathcal{P}(p)$ given by vector $p=\left(p_{i_{1}}=\right.$ $\left.p_{1}=1, p_{i_{2}} \ldots, p_{i_{k}}\right)$ with cuts at $c_{i_{j}}\left(p_{i_{j}}\right)$ in $J_{H}^{i_{j}}$, where the first cut comes at $c_{i_{1}}\left(p_{i_{1}}\right)=$ $c_{1}\left(p_{1}\right)=c_{1}(1)=a_{H}^{1}$, corresponding to $i_{1}=1$.

Proof of Theorem 11 (Optimal Pure Titles): From Lemma 5 (Cuts) we can already assume that all the cuts are in the $J_{H}^{i}$, and that no $J_{H}^{i}$ has more than one cut. If any $J_{H}^{i}$ had no cuts, then the status incentive to work of employees of type $i$ would be 0 .

Proof of Corollary to Theorem 11 See the proof of Theorem 1b in DubeyGeanakoplos (2010).

Proof of Theorem 12 (Optimal Wage-Title Schedule with Secret Wages and Risk Neutrality) In view of Theorem 11, if there is exactly one cut per type, we may identify the partition $\mathcal{P}(p)$ with the probabilities $p=\left(p_{1}, \ldots, p_{\ell}\right)$, where $p_{1}=1$. The number $p_{i}$ is the probability with which an employee who does put in effort gets a title corresponding to those of his ability class who do work. To the extent $p_{i}<1$, the incentive of type $i$ employees to work is reduced. On the other hand, when $p_{i}<1$, the status incentive of employees of type $i-1$ is enhanced, because by working then can come equal in status with a fraction $1-p_{i}$ of the workers of type i. Every ability type $i \geq 2$ has a substantial status incentive to work because shirking forces them to be classified with the type $i-1$ just below them. But the lowest ability type $i=1$ does not have that incentive.

Suppose there are $N_{1}, \ldots N_{\ell}$ employees of type $i=1, \ldots, \ell$. Given the title partition $p=\left(p_{1}, \ldots, p_{\ell}\right)$, the status incentive to work for the $\ell$ types is

$$
\begin{aligned}
I^{1}(p) & =p_{1}\left[\left(N_{1}-1\right)+\left(1-p_{2}\right) N_{2}\right] \\
I^{i}(p) & =p_{i}\left[\left(N_{i}-1\right)+p_{i-1} N_{i-1}+\left(1-p_{i+1}\right) N_{i+1}\right] \text { for } 2 \leq i \leq \ell-1 \\
I^{\ell}(p) & =p_{\ell}\left[\left(N_{\ell}-1\right)+p_{\ell-1} N_{\ell-1}\right] .
\end{aligned}
$$

When working, an employee of type $2 \leq i \leq \ell-1$ might get unlucky, with probability $1-p_{i}$, and find himself no better off than if he shirked. But with probability $p_{i}$ he will be lucky, outranking the fraction $p_{i-1}$ of type $i-1$ he otherwise would be equal with, and coming equal with the fraction $1-p_{i+1}$ of type $i+1$ he would otherwise have lost out against. In addition, he either outranks (instead of equalling) or equals (instead of being outranked by) every employee of his own type. This gives the formula $I^{i}(p)$ for $2 \leq i \leq \ell-1$. Taking $N_{0}=N_{\ell+1}=0$ gives the formulas for $I^{1}(p)$ and $I^{\ell}(p)$.

Since the $I^{i}(p)$ are continuous, and since $[0,1]^{\ell}$ is compact, an optimal $\tilde{p}$ clearly exists. (Of course $\tilde{p}_{1}=1$, for why reward any employee of type 1 for shirking.) We begin by showing that Lemma 4 (Cuts) still applies when employees are also motivated by money. 
Lemma 6 (Cuts with Wages): Suppose there are $\ell$ disparate types, and an original wage-title schedule (with wages secret or public). Then the total incentive to work of every employee can be improved (or left unchanged) by another wage schedule whose wage bill is unchanged, together with a title partition with the lowest cut at $a_{H}^{1}$, and at most one cut in every $J_{H}^{i}, i \geq 1$, and no other cuts. Furthermore, if the original wage schedule was public (i.e. measurable with respect to the original titles partition), then the new wage schedule can also be taken to be public (i.e. measurable with respect to the new titles partition).

Proof of Lemma 6 (Cuts with Wages): Suppose there is at least one cut in the interval $\left(b_{H}^{i-1}, a_{H}^{i}\right)$. Take the topmost such cut and move it right to $a_{H}^{i}$. Set the wage for outputs between the old topmost cut and $a_{H}^{i}$ equal to the wage on the left of the old topmost cut, and leave all other wages the same. This restores the measurability of wages. Moreover, this does not raise the wage of any unilaterally deviant shirker of type $i$, nor does it lower the wage of the worker of type $i$. At the same time, the status of the deviant shirker stays the same or goes down, while the status of the worker of type $i$ stays the same. Thus the status incentive to work for type $i$ is also not hurt. By iteratively moving cuts in this manner, we may assume that there are no cuts in any of the intervals $\left(b_{H}^{i-1}, a_{H}^{i}\right)$.

From this point we can repeat the argument in the proof of Lemma 4 (Cuts) and show that in the new partition given there, the status incentive to work of every employee is improved (or held constant). If wages are secret, they need not change, and so total incentives have gone up or stayed the same. If wages are public, then the removal of the top cut in $J_{H}^{i}$, (as in the proof of Lemma 4) might require a change in the wage schedule to maintain measurability with respect to the titles partition. Replace the wages on the cells just below and above the removed cut by the average per capita wage over those two cells. This restores measurability of the wage schedule and leaves the wage bill unchanged. The expected wage of a worker of type $i$ stays the same or increases. Hence his expected utility of working must go up or stay the same by concavity of his utility. His wage if he shirks is unaffected, hence his wage incentive to work rises or stays the same. For any employee of type $j>i$, his wage if he shirks either stays the same (in which case his working wage does too) or falls. If his working wage wage falls at all, it must have been the same as his shirking wage, and must fall by the same amount (with probability at most 1). Hence his incentive to work cannot go down.

Lemma 6 (Cuts with Wages) already guarantees that we need only consider at most one cut per $J_{H}^{i}$. To allow for the possibility of missing cuts, we consider the k-vector $p=\left(p_{i_{1}}=p_{1}=1, p_{i_{2}} \ldots, p_{i_{k}}\right)$, where the length $\mathrm{k}$ can vary. This defines the partition $\mathcal{P}(p)$ with cuts at $c_{i_{j}}\left(p_{i_{j}}\right)$ in $J_{H}^{i_{j}}$, where the first cut comes at $a_{H}^{1}$, corresponding to $i_{1}=1$. Let $I^{i}(p)$ denote the status incentive created by the title partition $p$ for employee-type $i$. Since wages are secret, they can be set independently of the title partition $p$. But then, on account of the concavity of $u$ and the riskneutrality of the owner, we might as well take wages to be a constant $w_{i}$ on $J_{H}^{i}$ and 
as low as possible (while respecting the constraint of monotonicity) on $J_{L}^{i}$, namely ${ }^{35}$ $w_{i-1}$. Thus the owner's optimization problem may be written

$$
\begin{gathered}
\min _{p, w} \sum_{i=1}^{\ell} w_{i} N_{i} \\
\text { s.t. } I^{i}(p)+u\left(w_{i}\right)-u\left(w_{i-1}\right) \geq d_{i}, \text { for } 1 \leq i \leq \ell \\
0 \leq p_{i_{j}} \leq 1, \text { for } 2 \leq j \leq k \\
1=i_{1} \leq i_{2} \leq \ldots i_{k} \leq \ell \\
w_{\min }=w_{0} \leq w_{1} \leq \ldots . \leq w_{l}
\end{gathered}
$$

Conclusion of the Proof of Theorem 12: As was said before, Lemma 6 (Cuts with Wages) already guarantees that we need only consider at most one cut per $J_{H}^{i}$, defined by the vector $\left(p_{i_{1}}=p_{1}=1, p_{i_{2}} \ldots, p_{i_{k}}\right)$ giving rise to cuts $c_{i_{j}}\left(p_{i_{j}}\right)$ in $J_{H}^{i_{j}}$, where the first cut comes at $a_{H}^{1}$, corresponding to $i_{1}=1$

Define the expected number of people $v_{i_{j}}$ in the region $V_{i_{j}}$ from each cut $c_{i_{j}}$ to the next cut $c_{i_{j}+1}$ (assuming everybody works) by

$$
\nu_{i_{1}}=N_{1}+N_{2}+\ldots+N_{i_{2}-1}+\left(1-p_{i_{2}}\right) N_{i_{2}}
$$

and, for $2 \leq j \leq k-1$

$$
\nu_{i_{j}}=p_{i_{j}} N_{i_{j}}+N_{i_{j}+1}+\ldots+N_{i_{j+1}-1}+\left(1-p_{i_{j}+1}\right) N_{i_{j+1}}
$$

and, finally

$$
\nu_{i_{k}}=p_{i_{k}} N_{i_{k}}+N_{i_{k}+1}+\ldots+N_{i_{\ell}-1}+N_{i_{\ell}}
$$

Suppose now that we eliminate all the cuts except the one at $a_{H}^{1}$. The status payoff of the shirker and the worker of type $j \notin\left\{i_{1}=1, i_{2}, \ldots, i_{k}\right\}$ is unchanged.

The status payoff of the shirker of type 1 is unchanged, but the status payoff (and hence the incentive) of the worker of type 1 goes up by the expected number of workers above cut $c_{i_{2}}$

$$
\nu_{i_{2}}+\nu_{i_{3}}+\ldots+\nu_{i_{k}}
$$

since now when a type 1 employee works, he comes equal with all these other people.

For any employee of type $i_{j} \in\left\{i_{2}, \ldots, i_{k}\right\}$, the status incentive after the cuts are removed is zero! Prior to the removal, the status incentive of $i_{j}$ was

$$
p_{i_{j}}\left(\nu_{i_{j-1}}+\nu_{i_{j}}-1\right)
$$

because when an employee of type $i_{j}$ worked, with probability $\left(1-p_{i_{j}}\right)$ he ended up with the same status as a shirker, and with probability $p_{i_{j}}$ he gained status by

\footnotetext{
${ }^{35}$ Consider any weakly monotonic wage function $w^{\prime}$. Let $w_{i}$ denote the average value of $w^{\prime}$ on $J_{H}^{i}$ and replace $w^{\prime}$ by the step function $w=\left(w_{0}, w_{1}, \ldots, w_{l}\right)$ as discussed. Then $w$ will not raise the expected wage bill, and will create no less wage incentives, compared to $w^{\prime}$.
} 
outranking all the people in region $V_{i_{j}-1}$ and coming equal with all the people in region $V_{i_{j}}$ (not counting himself). Thus the loss in status incentive is $p_{i_{j}}\left(\nu_{i_{j-1}}+\nu_{i_{j}}-1\right)$.

Given these changes in status incentives, it is possible to change the wages, in fact to lower the total wage bill, and yet leave all the employees with the same total incentive (i.e., status incentive plus wage incentive). First, recall that for outputs below $c_{i_{1}}=c_{1}=a_{H}^{1}$, the wage is at $w_{\text {min }}$. For outputs above $c_{i_{1}}$ lower all wages by $\nu_{i_{2}}+\nu_{i_{3}}+\ldots+\nu_{i_{k}}$. This restores the original total incentive of all employees of types below $i_{2}$ and continues to leave unchanged the total incentive of each types $j \notin\left\{i_{1}=1, i_{2}, \ldots, i_{k}\right\}$. By assumption, the resulting wages must still be strictly above $w_{\min }$. Otherwise, the employees of type 1 would now be incentivized to work without a positive wage incentive (or indeed despite a negative wage incentive) contradicting our assumption that status incentive alone can never overcome the disutility of working).

For outputs above $c_{i_{2}}$, now raise all wages by $\nu_{i_{1}}+\nu_{i_{2}}-1<\nu_{i_{1}}+\nu_{i_{2}}$. This restores the total incentive of employees of type $i_{2}$ and leaves unchanged all other incentives. Successively raise all wages for outputs above $c_{i_{j}}$ by $\nu_{i_{j-1}}+\nu_{i_{j}}-1<\nu_{i_{j-1}}+\nu_{i_{j}}$. As before, this restores the total incentive of employees of type $i_{j}$ without changing any other incentives. Thus the new wage schedule gives all employees precisely the same total incentive as before.

We now show that the new wage schedule has a smaller total wage than the original. We compute the change in the wage bill by multiplying the number of workers by the change in their wages. The change in the total wage bill is thus strictly less than

$$
\begin{aligned}
& -\left(\nu_{i_{1}}+\nu_{i_{2}}+\nu_{i_{3}}+\ldots+\nu_{i_{k}}\right)\left[\nu_{i_{2}}+\nu_{i_{3}}+\ldots+\nu_{i_{k}}\right] \\
& +\left(\nu_{i_{2}}+\nu_{i_{3}}+\ldots+\nu_{i_{k}}\right)\left[\nu_{i_{1}}+\nu_{i_{2}}\right] \\
& +\left(\nu_{i_{3}}+\ldots+\nu_{i_{k}}\right)\left[\nu_{i_{2}}+\nu_{i_{3}}\right]+\ldots \\
& +\left(\nu_{i_{k}}\right)\left[\nu_{i_{k-1}}+\nu_{i_{k}}\right] \\
& =0
\end{aligned}
$$

This shows that the original wage-title schedule can be strictly improved by another wage-title schedule in which the title partition has just one cut at $a_{H}^{1}$.

But given a title partition with just one cut at $a_{H}^{1}$, it is evident (in view of our Necessity-of-Wages Assumption) that the optimal secret wage schedule is as stated.

Proof of Theorem 13 (Exorbitant Elite Wages): Recall that the owner seeks to minimize his wage bill, subject to incentivizing every employee to work. In light of Lemma 6 (Cuts with Wages), we may restrict attention to title partitions with at most one cut in every $J_{H}^{i}$. It is easy to see that every such cut must occur. For if some $J_{H}^{i}$ had no cut, then $J_{L}^{i}$ and $J_{H}^{i}$ would have the same title, hence employees of type $i$ would have no status incentive to work; but then, since wages are public, wages would have to be constant across $J_{L}^{i}$ and $J_{H}^{i}$, and then employees of type $i$ would have no wage incentive either. Therefore the title partition is represented by the full vector $p=\left(p_{1}, p_{2}, \ldots, p_{\ell}\right)$. Denote by $\mathcal{W}\left(p_{1}, p_{2}, \ldots, p_{\ell}\right)$ the class of wage schedules that are measurable with respect to the title partition $\mathcal{P}\left(p_{1}, p_{2}, \ldots, p_{\ell}\right)$. One critical aspect 
of the problem is that we have capped the maximum wage at an arbitrary, but high, level $M .^{36}$

We may then state the employer's optimization problem as follows:

$$
\begin{gathered}
\min _{p, w} \sum_{i=1}^{\ell}\left[\left(1-p_{i}\right) w_{i-1}+p_{i} w_{i}\right] N_{i}=\min _{p, w}\left\{\sum_{i=1}^{\ell} w_{i-1} N_{i}+\sum_{i=1}^{\ell} p_{i}\left(w_{i}-w_{i-1}\right) N_{i}\right\} \\
\text { s.t. } \tilde{I}_{i} \equiv I^{i}(p)+p_{i}\left(u\left(w_{i}\right)-u\left(w_{i-1}\right)\right) \geq d_{i}, \text { for } 1 \leq i \leq \ell \\
0 \leq p_{i} \leq 1 \text { and } w \in \mathcal{W}\left(p_{1}, p_{2}, \ldots, p_{\ell}\right) \\
w_{\min }=w_{0} \leq w_{1} \leq \cdots \leq w_{\ell} \leq M
\end{gathered}
$$

The total incentive, $\tilde{I}_{i}$, of each agent of type $i$ consists, as before, of the status incentive $I_{i} \equiv I^{i}(p)$ plus a wage incentive $p_{i}\left(u\left(w_{i}\right)-u\left(w_{i-1}\right)\right)$.

It will be useful to keep in mind throughout that raising $p_{i}$ has the effect of raising $\tilde{I}_{i}$ and $\tilde{I}_{i+1}$ and lowering $\tilde{I}_{i-1}$ without disturbing other incentives. Similarly, raising $w_{i}$ raises $\tilde{I}_{i}$ and lowers $\tilde{I}_{i+1}$, with no other effect.

We shall show inductively, starting with $i=1$, that $p_{i}=1$ for all $i=1, \ldots, \ell-1$. First note that, thanks to the Necessity of Wages Assumption, $p_{1}>0$ and $w_{1}>w_{0}$. Suppose $p_{1}<1$. Define $\tilde{w}_{1}$ by $\tilde{w}_{1}=\left(1-p_{1}\right) w_{0}+p_{1} w_{1}$. Let the employer raise $p_{1}$ to 1 and lower $w_{1}$ to $\tilde{w}_{1}$, leaving all other $p_{i}$ and $w_{i}$ unchanged. Clearly this does not affect the wage bill. At the same time the status incentive of type 1 does not go down (indeed it goes up, unless $N_{1}=1$ and $p_{2}=1$ when it remains the same); both the status incentive and the wage incentive of type 2 go up (the first on account of the rise in $p_{1}$, and the second on account of the fall in $w_{1}$ ); the incentives of players of type $3, \ldots, l$ are undisturbed; and the wage incentive of type 1 does not go down (indeed it goes up if $u$ is strictly concave) as the following calculation shows:

$$
u\left(\tilde{w}_{1}\right)-u\left(w_{0}\right) \geq\left(1-p_{1}\right) u\left(w_{0}\right)+p_{1} u\left(w_{1}\right)-u\left(w_{0}\right)=p_{1}\left[u\left(w_{1}\right)-u\left(w_{0}\right)\right]
$$

To sum up, the employer's maneuver improves both status and wage incentives of type 2, without hurting any other incentives and without raising the wage bill. Next, the employer can decrease $w_{2}$ by a small $\epsilon$, and thus lower the wage bill. The decrease of $w_{2}$ has just two effects on incentives: it raises the wage incentive of type 3 and lowers the wage incentive of type 2 and, other than this, has no effect on any other wage or status incentives (including the status incentive of type 2). For small enough $\varepsilon$, the incentive of type 2 will not fall below his original (pre-maneuver) level. Thus the principal does better, generating incentives that are no worse, for a lower wage bill, a contradiction. We conclude that $p_{1}=1$.

Inductively assume that $p_{1}=\cdots=p_{i-1}=1$ for $i<\ell$. If $p_{i}<1$, we shall reach a contradiction by finding a cheaper way of providing the same incentives.

In what follows we shall be making small changes in wages to get from $w_{j}$ to $\tilde{w}_{j}$,i.e., $\left|w_{j}-\tilde{w}_{j}\right|<\kappa \varepsilon$ for some constant $\kappa$ and infinitesimal $\varepsilon$.So, denoting the

\footnotetext{
${ }^{36}$ This helps to keep the problem compact. The bound $M$ may also be interpreted as the degree of inequity aversion in the society (see Fehr-Schmidt (1999)).
} 
derivative $u^{\prime}\left(w_{j}\right)=\lambda_{j}$, we shall write $^{37} u\left(w_{j}\right)-u\left(\tilde{w}_{j}\right)=\lambda_{j}\left(w_{j}-\tilde{w}_{j}\right)$ (see the Differentiability Assumption). Note that $\lambda_{1} \geq \lambda_{2} \geq \ldots . . \geq \lambda_{\ell}$ since $u$ is concave and since $w_{1} \leqslant w_{2} \leqslant \ldots \leqslant w_{\ell}$.

Set $\tilde{p}_{i}=p_{i}+\varepsilon$ and set $\tilde{w}_{i-1}$ to satisfy $\lambda_{i-1}\left(\tilde{w}_{i-1}-w_{i-1}\right)=\varepsilon N_{i}$, i.e., $\tilde{w}_{i-1}=$ $w_{i-1}+\lambda_{i-1}^{-1} \varepsilon N_{i}$. Then the status incentive of $i-1$ goes down by $\varepsilon N_{i} p_{i-1}=\varepsilon N_{i}$, but his wage incentive goes up by the same amount: since $p_{i-1}=1$, we have $p_{i-1}\left(u\left(\tilde{w}_{i-1}\right)-\right.$ $\left.u\left(w_{i-2}\right)\right)=u\left(w_{i-1}\right)-u\left(w_{i-2}\right)+u\left(\tilde{w}_{i-1}\right)-u\left(w_{i-1}\right)=u\left(w_{i-1}\right)-u\left(w_{i-2}\right)+\lambda_{i-1}\left(\lambda_{i-1}^{-1} \varepsilon N_{i}\right)$ $=p_{i-1}\left(u\left(w_{i-1}\right)-u\left(w_{i-2}\right)\right)+\varepsilon N_{i}$.

Also, the status incentive of $i$ goes up by

$$
\Delta I_{i}(\varepsilon) \equiv \varepsilon\left[\left(N_{i}-1\right)+\left(1-p_{i+1}\right) N_{i+1}+N_{i-1}\right] .
$$

This allows us to reduce his wage incentive by the same amount. So, set $\tilde{w}_{i}$ to satisfy $\tilde{p}_{i}\left[u_{i}\left(\tilde{w}_{i}\right)-u_{i}\left(\tilde{w}_{i-1}\right)\right]-$

$$
\begin{aligned}
\tilde{p}_{i} \lambda_{i}\left(\tilde{w}_{i}-\tilde{w}_{i-1}\right) & \equiv p_{i} \lambda_{i}\left(w_{i}-w_{i-1}\right)-\Delta I_{i}(\varepsilon), \text { i.e. }, \\
\tilde{p}_{i}\left(\tilde{w}_{i}-\tilde{w}_{i-1}\right) & \equiv p_{i}\left(w_{i}-w_{i-1}\right)-\frac{\Delta I_{i}(\varepsilon)}{\lambda_{i}}
\end{aligned}
$$

For small $\varepsilon, \Delta I_{i}(\varepsilon)$ is small, so $p_{i}\left(w_{i}-w_{i-1}\right)>0$ implies that $\tilde{p}_{i}\left(\tilde{w}_{i}-\tilde{w}_{i-1}\right)>0$, which in turn implies $\tilde{w}_{i}>\tilde{w}_{i-1}$, retaining the monotonicity of the revised wages. We shall be assuming $\varepsilon$ small enough to guarantee monotonicity in all future wage revisions, without explicitly saying so.

Note that, since $w_{i}>w_{i-1}$ and $p_{i}+\varepsilon<1$ (if $p_{i}<1$ and $\varepsilon$ is small) and $\lambda_{i-1}>\lambda_{i}$ and $N_{i-1} \geq 1$,

$$
\begin{aligned}
\tilde{w}_{i} & =\frac{p_{i}}{\tilde{p}_{i}} w_{i}+\tilde{w}_{i-1}-\frac{p_{i}}{\tilde{p}_{i}} w_{i-1}-\frac{1}{\lambda_{i} \tilde{p}_{i}} \Delta I_{i}(\varepsilon) \\
& =\frac{p_{i}}{\tilde{p}_{i}} w_{i}+w_{i-1}+\frac{\varepsilon N_{i}}{\lambda_{i-1}}-\frac{p_{i}}{\tilde{p}_{i}} w_{i-1}-\frac{1}{\lambda_{i} \tilde{p}_{i}} \Delta I_{i}(\varepsilon) \\
& \leq \frac{p_{i}}{p_{i}+\varepsilon} w_{i}+\left(1-\frac{p_{i}}{p_{i}+\varepsilon}\right) w_{i-1}+\frac{1}{\lambda_{i}}\left(\varepsilon N_{i}-\frac{1}{p_{i}+\varepsilon} \Delta I_{i}(\varepsilon)\right) \\
& <w_{i}+\frac{1}{\lambda_{i}}\left(\varepsilon N_{i}-\varepsilon\left[\left(N_{i}-1\right)+\left(1-p_{i+1}\right) N_{i+1}+N_{i-1}\right]\right) \\
& \leq w_{i}-\frac{1}{\lambda_{i}}\left(\varepsilon\left(N_{i-1}-1\right)\right) \leq w_{i}
\end{aligned}
$$

Finally, the status incentive of $i+1$ goes up by

$$
\Delta I_{i+1}(\varepsilon) \equiv \varepsilon p_{i+1} N_{i}
$$

\footnotetext{
${ }^{37}$ This to be understood as a first-order approximation, ignoring all higher-order effects. Strictly speaking we should replace $\lambda_{j}$ with a number between $u^{\prime}\left(w_{j}\right)$ and $u^{\prime}\left(\tilde{w}_{j}\right)$. But the reader may easily check that our argument below holds, mutatis mutandis, with these strictly correct $\lambda^{\prime} s$ in place of ours (to represent exactly, rather than approximately, the changes in wage-utilities).
} 
Therefore the wage incentive of $i+1$ can be reduced by the same amount. So set $\tilde{w}_{i+1}$ to satisfy

$$
\begin{aligned}
p_{i+1} \lambda_{i+1}\left(\tilde{w}_{i+1}-\tilde{w}_{i}\right) & =p_{i+1} \lambda_{i+1}\left(w_{i+1}-w_{i}\right)-\Delta I_{i+1}(\varepsilon), \text { i.e. } \\
p_{i+1}\left(\tilde{w}_{i+1}-\tilde{w}_{i}\right) & =p_{i+1}\left(w_{i+1}-w_{i}\right)-\frac{\Delta I_{i+1}(\varepsilon)}{\lambda_{i+1}}
\end{aligned}
$$

Since $\tilde{w}_{i}<w_{i}$, clearly $\tilde{w}_{i+1}<w_{i+1}$. Hence recursively setting

$$
\tilde{w}_{j}-\tilde{w}_{j-1}=w_{j}-w_{j-1} \text { for } j>i+1
$$

further lowers wages without changing incentives.

It remains to show that the wage bill defined in the owner minimization problem has gone down. The only terms that increase are

$$
w_{i-1} N_{i} \text { and } p_{i-1}\left(w_{i-1}-w_{i-2}\right) N_{i-1}
$$

while many terms are reduced, including

$$
p_{i}\left(w_{i}-w_{i-1}\right) N_{i} \text { and } p_{i+1}\left(w_{i+1}-w_{i}\right) N_{i+1} .
$$

The increases add up to

$$
\frac{1}{\lambda_{i-1}}\left(\varepsilon N_{i} N_{i}+\varepsilon N_{i} N_{i-1}\right),
$$

while just these two reductions add to

$$
\begin{aligned}
& \frac{1}{\lambda_{i}} \Delta I_{i}(\varepsilon) N_{i}+\frac{1}{\lambda_{i+1}} \Delta I_{i+1}(\varepsilon) N_{i+1} \geq \frac{1}{\lambda_{i-1}}\left(\Delta I_{i}(\varepsilon) N_{i}+\Delta I_{i+1}(\varepsilon) N_{i+1}\right) \\
& =\frac{1}{\lambda_{i-1}}\left(\varepsilon N_{i}\left[\left(N_{i}-1\right)+\left(1-p_{i+1}\right) N_{i+1}+N_{i-1}\right]+\varepsilon p_{i+1} N_{i} N_{i+1}\right) \\
& =\frac{1}{\lambda_{i-1}}\left(\varepsilon N_{i}^{2}+\varepsilon N_{i} N_{i-1}+\varepsilon N_{i}\left(N_{i+1}-1\right)\right) .
\end{aligned}
$$

( the inequality following from the fact that $\lambda_{i-1} \geq \lambda_{i} \geq \lambda_{i+1}$ on account of the concavity of $u$ ). Since $N_{i+1} \geq 1$, the reduction is at least as big as the increase. But we have ignored many other strictly positive reductions (for example in $w_{i+1} N_{i+1}$ ). This contradiction proves that $p_{i}=1$, for $i=2, \ldots, \ell-1$ and establishes part (a) of the theorem.

Now suppose $w_{\ell}<M$. Since we assumed $p_{\ell}\left(w_{\ell}-w_{\ell-1}\right)>0$, clearly $p_{\ell}>0$. Lower $p_{\ell}$ by $\varepsilon$. This raises the status incentive of type $\ell-1$ workers by $\varepsilon N_{\ell}$, enabling us to lower the wage incentive for type $\ell-1$ by the same amount.

Recalling that $p_{\ell-1}=1$, and (by the Asymptotic Risk Neutrality Assumption) $w_{\ell-2}>w^{\tau}$ so that $\lambda_{\ell-1}=\lambda_{\ell}=\lambda$, set $\tilde{w}_{\ell-1}$ to satisfy

$$
\left(\tilde{w}_{\ell-1}-w_{\ell-2}\right)=\left(w_{\ell-1}-w_{\ell-2}\right)-\frac{\varepsilon N_{\ell}}{\lambda} .
$$


This drop in $p_{\ell}$ unfortunately lowers the status incentive of type $\ell$ by $\varepsilon\left(N_{\ell}-1+\right.$ $\left.N_{\ell-1}\right)$. Therefore we must raise the wage incentive of $\ell$, choosing $\tilde{w}_{\ell}$ to solve

$$
\left(p_{\ell}-\varepsilon\right)\left(\tilde{w}_{\ell}-\tilde{w}_{\ell-1}\right)=p_{\ell}\left(w_{\ell}-w_{\ell-1}\right)+\frac{\varepsilon\left(N_{\ell-1}+N_{\ell}-1\right)}{\lambda} .
$$

Fortunately, there is no group $\ell+1$ to be affected by the change in $p_{\ell}$, which is why it will turn out to be optimal to lower $p_{\ell}$ as long as $w_{\ell}<M$, whereas it was shown to be optimal to raise $p_{i}$ all the way to 1 for any $i<\ell$.

Indeed the terms in the wage bill that change are

$$
w_{\ell-1} N_{\ell}+p_{\ell-1}\left(w_{\ell-1}-w_{\ell-2}\right) N_{\ell-1}+p_{\ell}\left(w_{\ell}-w_{\ell-1}\right) N_{\ell} .
$$

The net change in those terms, by our estimates above, is

$$
\begin{aligned}
& \frac{1}{\lambda}\left(-\varepsilon N_{\ell}^{2}-\varepsilon N_{\ell} N_{\ell-1}+\varepsilon\left(N_{\ell-1}+N_{\ell}-1\right) N_{\ell}\right) \\
& =-\frac{1}{\lambda} \varepsilon N_{\ell}<0
\end{aligned}
$$

showing that the wage bill can be reduced, a contradiction. This proves that $w_{\ell}=M$.

Having proved that $p_{i}=1$ for any $1 \leq i \leq \ell-1$, it follows that the status incentives for $1 \leq i \leq \ell-2$ are given by $I_{i}=I_{\ell}^{i}\left(1, \ldots, 1, p_{\ell}\right)=N_{i}+N_{i-1}-1$. Hence the wages are recursively determined (starting from $w_{0}=w_{\min }$ ) for $1 \leq i \leq \ell-2$ by the equation

$$
u\left(w_{i}\right)-u\left(w_{i-1}\right)=d_{i}-I_{i}=d_{i}-\left(N_{i}+N_{i-1}-1\right) .
$$

Next, it will be convenient to scale the money by $\lambda$. Accordingly denote $w_{j}^{*}=\lambda w_{j}$, $M^{*}=\lambda M$ etc. Then we also have

$$
w_{\ell-1}^{*}-w_{\ell-2}^{*}=d_{\ell-1}-\left[N_{\ell-2}+N_{\ell-1}+\left(1-p_{\ell}\right) N_{\ell}-1\right]
$$

and $\epsilon$

$$
M^{*}-w_{\ell-1}^{*}=\frac{d_{\ell}}{p_{\ell}}-\left[N_{\ell-1}+N_{\ell}-1\right]
$$

(recalling that $w_{\ell} \equiv M$,hence $w_{\ell}^{*} \equiv M^{*}$, in the last equation). We now show that there is a unique solution $w_{\ell-1}^{*}, p_{\ell}$ of these two simultaneous equations, so that the optimal wage schedule is determined uniquely. To do this, we add the two equations to get a convex quadratic in the single unknown $p_{\ell}$. We then show that it has a positive value at $p_{\ell}=0$ and a negative value at $p_{\ell}=1$ and therefore a unique solution $p_{\ell}$ in $(0,1)$.

More precisely, multiplying each equation by $p_{\ell}$ and then adding them yields

$$
-p_{\ell}\left(M^{*}-w_{\ell-2}^{*}-d_{\ell-1}\right)-p_{\ell}\left(N_{\ell-2}+N_{\ell-1}-1\right)-N_{\ell} p_{\ell}\left(1-p_{\ell}\right)+d_{\ell}-p_{\ell}\left(N_{\ell-1}+N_{\ell}-1\right)=0
$$

i.e. $f\left(p_{\ell}\right)=0$, say. Clearly $f(0)=d_{\ell}>0$. Also, since $\lambda w_{i}-\lambda w_{i-1} \leq u\left(w_{i}\right)-u\left(w_{i-1}\right) \leqslant$ $d_{i}$ for $1 \leq i \leq \ell-1$ ( the first inequality following from the concavity of $u$, the second from the fact wage incentive plus title incentive equals disutility), we have

$$
w_{i}^{*} \leq w_{\min }^{*}+d_{1}+\ldots+d_{i}
$$


for all $1 \leq i \leq \ell-1$, and in particular for $i=\ell-2$. This, in conjunction with $M^{*}>d_{1}+\ldots+d_{\ell}$ and $N_{i} \geq 1$, implies $f(1)<0$.

Finally, since the wage incentive of $\ell$ is at most $d_{\ell}$, we have

$$
p_{\ell}\left(M^{*}-w_{\ell-1}^{*}\right) \leq d_{\ell}
$$

hence

$$
p_{\ell} \leq \frac{d_{\ell}+p_{\ell} w_{\ell-1}^{*}}{M^{*}} \leq \frac{d_{\ell}+w_{\ell-1}^{*}}{M^{*}} \leq \frac{w_{\min }^{*}+\sum_{i=1}^{\ell} d_{i}}{M^{*}}
$$

Proof of Corollary to Theorem 12: The incentive formulae are trivially generated by plugging $p_{i}=1$ for $1 \leq i \leq \ell-1$ into the status incentives for each agent, and by observing that $p_{\ell} \leq\left[w_{\min }+\sum_{i=1}^{\ell} d_{i}\right] / M \approx 0$ if $M$ is large.

The wage differentials were explicitly computed in the proof of Theorem 12 .

Proof of Theorem 12' (Variant of Theorem 12 for Incomplete Information) Define $\nu_{i_{j}}$ just as in the proof of Theorem 12 but with each $N_{i}$ replaced by $\theta_{i} N$ (i.e., the expected number of employees in the region $\left.V_{i_{j}}\right)$ and define $\nu_{i_{j}}^{*}=\theta_{i}(N-1)=$ $[(N-1) / N] \nu_{i_{j}}$ ( the expected number of others in $V_{i_{j}}$, conditional on one employee - of any type - standing aside). Then re-read the proof of Theorem 12 with the following amendments: the changes in status incentives are given by the same formulae replacing $\nu_{i_{j}}$ by $\nu_{i_{j}}^{*}$ throughout and dropping " -1 " ( thus $\nu_{i_{1}}+\nu_{i_{2}}-1$ is replaced by $\nu_{i_{1}}^{*}+\nu_{i_{2}}^{*}$, etc.). Repeating the maneuver of wage changes as we move to the single cut at $a_{H}^{1}$, the total change in wage bill is no more than

$$
\begin{aligned}
& -\left(\nu_{i_{1}}+\nu_{i_{2}}+\nu_{i_{3}}+\ldots+\nu_{i_{k}}\right)\left[\nu_{i_{2}}^{*}+\nu_{i_{3}}^{*}+\ldots+\nu_{i_{k}}^{*}\right] \\
& +\left(\nu_{i_{2}}+\nu_{i_{3}}+\ldots+\nu_{i_{k}}\right)\left[\nu_{i_{1}}^{*}+\nu_{i_{2}}^{*}\right] \\
& +\left(\nu_{i_{3}}+\ldots+\nu_{i_{k}}\right)\left[\nu_{i_{2}}^{*}+\nu_{i_{3}}^{*}\right]+\ldots \\
& +\left(\nu_{i_{k}}\right)\left[\nu_{i_{k-1}}^{*}+\nu_{i_{k}}^{*}\right]
\end{aligned}
$$

But since $\nu_{i_{j}}^{*}=[(N-1) / N] \nu_{i_{j}}$ we may undo the stars in the above display (scaling the expression by $(N-1) / N)$, which reveals that the displayed expression is 0 as before. though it may no longer be the unique optimal schedule (as was the case with complete information)

Proof of Theorem 13' (Variant of Theorem 13 for Incomplete Information) Let us outline the changes needed in the proof of Theorem 12 for establishing this variant. Notice first that we must once again have exactly one cut in each interval $J_{H}^{i}$, for if such a cut were missing then wages would have to be the same for $J_{L}^{i}$ and $J_{H}^{i}$ as these two intervals get the same title; and thus $i$ would have no incentive to work whatsoever. Now, as pointed out earlier, the formulae for wage bill (resp. status incentive) are preserved if we replace $N_{i}\left(\operatorname{resp} . N_{i}\right.$ and $\left.N_{i}-1\right)$ by $\bar{N}_{i}=\theta_{i} N$ 
(resp. $\left.N_{i}^{*}=\theta_{i}(N-1)=[(N-1) / N] \bar{N}_{i}\right)$. With these substitutions we can literally repeat the proof of Theorem 11; indeed, the estimates for changes in the wage bill, as we go through the wage-schedule modifications prescribed in that proof, will be the same exact expressions as before, replacing $N_{i} N_{i-1}$ (or, $N_{i} N_{i+1}$ ) in the proof by $[(N-1) / N] \bar{N}_{i} \bar{N}_{i-1}$ (or, $\left.[(N-1) / N] \bar{N}_{i} \bar{N}_{i+1}\right)$ throughout. The reason is that changes in the wage bill are the product of two terms:

(a) changes in the wage (which compensate for changes in status incentive, and therefore involve terms $N_{i}^{*}$ ); and

(b) the expected number of workers for whom that change is occuring ( which involve $\bar{N}_{i}$ )

But products like $\bar{N}_{i} N_{i-1}^{*}$ are equal to $[(N-1) / N] \bar{N}_{i} \bar{N}_{i-1}$. This summarizes the main changes, and the rest of the argument proceeds exactly as before. It shows that positive reductions are achieved in the wage bill whenever we increase $p_{i}<1$ to $p_{i}+\varepsilon$ for $1 \leq i \leq l-1$, hence such $p_{i}=1$ as claimed; and when we lower $p_{l}$, the wage bill is unaffected (instead of being strictly reduced), establishing the claim regarding $p_{l}$ and $w_{l}$. 